Research Article

\title{
Experimental Centrifuge Study of the Effects of Valley Topography on the Behavior of a Concrete Face Rockfill Dam
}

\author{
Chao Tian $\left(\mathbb{D},{ }^{1}\right.$ Xuedong Zhang, ${ }^{2}$ and Lifeng Wen ${ }^{1}$ \\ ${ }^{1}$ State Key Laboratory of Eco-Hydraulics in Northwest Arid Region, Xi'an University of Technology, Xi'an 710048, China \\ ${ }^{2}$ China Institute of Water Resources and Hydropower Research, Beijing 100044, China \\ Correspondence should be addressed to Chao Tian; 1170411026@stu.xaut.edu.cn
}

Received 8 September 2021; Accepted 2 October 2021; Published 21 October 2021

Academic Editor: Shengwen Tang

Copyright (c) 2021 Chao Tian et al. This is an open access article distributed under the Creative Commons Attribution License, which permits unrestricted use, distribution, and reproduction in any medium, provided the original work is properly cited.

The recent focus on water conservancy projects globally has resulted in the construction of increased numbers of concrete face rockfill dams in narrow valleys. However, valley topography impacts the deformation of a dam and further influences the distribution of stress and position of cracks on the face slab. This study conducted two centrifuge experiments to study the influence of the valley topography on the behavior of a concrete face rockfill dam from construction to impoundment. Experimental models of concrete face slab sand-gravel dams with "U"-type and "V"-type valley topographies were established. The settlement of the dam crest, the displacement of the upstream slope of the dam, and the stress on both sides of the face slab were observed. The experiment also represented the cracking of the face slab during impoundment. The results showed that the "V"type valley topography effectively reduced the progression of dam crest settlement and influenced stress on the slab resulting from impounded water pressure. Furthermore, the flexural form of the face slab in the " $U$ "-type valley topography took on a " $D$ " shape and cracks progressively developed on the face slab with increased water load. The flexural form of the face slab in the "V"-type valley topography showed a "B" shape, and cracks occurred under a particular water impoundment pressure.

\section{Introduction}

Concrete face rockfill dams are widely constructed globally due to their low cost and easy construction. A concrete face rockfill dam is composed of rockfill and concrete slabs placed on the upstream side, with well-compacted rockfill playing a key function in resisting the upstream impoundment load and the concrete slabs with waterstop structures preventing the leakage of impounded water downstream [1]. The design code and general guidelines have allowed the plane strain method to be used in the construction of a concrete face rockfill dam. This simplified method can achieve good results for a wide and shallow valley topography $[2,3]$. However, the recent focus on water conservancy projects has resulted in the construction of increased numbers of concrete face slab sand-gravel dams in narrow valleys in Western China. The plane strain method is unable to consider the effects of valley topography on the behavior of a concrete face rockfill dam. Valley topography has an appreciable impact on dam deformation behavior and the distribution of stress in the face slab. Therefore, there has been an increased focus on the influence of valley topography on concrete face rockfill dams in recent years.

There have been numerous studies on the influence of valley topography on the deformation of concrete face rockfill dams. Kirn and Sarkaria [4], Thomas [5], and Pinto and Marques [6] analyzed historical monitoring data for concrete face rockfill dams and found that the behavior of this type of dam is heavily dependent on valley topography. Giudici et al. [7] analyzed the correlation between the deformation of a concrete face rockfill dam and valley topography using historical monitoring data for 30 concrete face rockfill dams in seven countries. They found that the compression modulus of a rockfill dam has the strongest correlation with valley shape, thereby impacting the deformation of a rockfill dam. Johannesson [8] found that the maximum dam crest settlement shows a nonlinear relationship with the valley shape, although it tends to stabilize 
after the shape of the valley changes to a certain extent. Kim and Kim [9] analyzed the monitoring data for 30 concrete face rockfill dams using the backpropagation (BP) neural network and found that narrow valley topography effectively influences the settlement of the dam crest when the construction modulus is below a certain threshold. Znamensky et al. [10] investigated data for the deformation of concrete face rockfill dams and found that a narrow valley shape could reduce dam crest settlement. Sukkarak et al. [11] presented a series of finite element analyses to investigate the influence of the valley shape on dam deformation and found that the impact of the valley topography on dam crest settlement was obvious when the dam slope was steep.

However, an additional challenge facing concrete face rockfill dam systems is the effect of complex valley topography on the integrity of the concrete face slab due to the pressure of retained water on the concrete slab [12]. Cheng et al. [13] analyzed the influence of valley topography on stress placed on the concrete face slab using finite element modelling (FEM) and found that although valley topography restricts the deformation of the slab, stress in the slab is enhanced, resulting in cracks. Arici and Zel [14] compared the use of two-dimensional (2D) and three-dimensional (3D) dam models for investigating the performance of concrete face rockfill dams and found that the 3D model experienced less displacement and cracking of the face slab after the impounding stage compared to that of the $2 \mathrm{D}$ dam model. Dang et al. [15] investigated the influence of valley topography on high concrete face rockfill dams and found that valley topography had a clear effect on the position and region of high tensile stress on the concrete face slab. Yang et al. [16] investigated the deformation of the Dashixia concrete face rockfill dam under complex valley topography and found that the valley topography increased the deformation gradient and stress gradient of the face slab. Wang et al. [17] studied the influence of valley topography on the deformation and stress of a high concrete face rockfill dam and found that the stress and deformation gradient of the face slab increased exponentially when the valley widthheight ratio was less than 2. Karalar and Çavuşli $[18,19]$ used a WIPP-creep viscoplastic material model and a burgercreep viscoplastic material model to represent the concrete slab, rockfill materials, and foundation of a concrete face rockfill dam within an investigation of the deformation of the Ilısu Dam in Turkey. Gao et al. [20] examined monitoring data for 22 concrete face rockfill dams across eight countries and found a low correlation between valley factors and deformation of the face slab below a certain valley factor threshold, whereas abrupt changes to the slope angle aggravated deformation of the slab. Li et al. [21, 22] and Wen et al. $[23,24]$ examined historical deformation data for earth-rockfill dams and found that valley topography has a more obvious influence on deformation of the face slab for high concrete face rockfill dams.

The deformation of the dam and the stress placed on the face slab are the most important risks facing the safety of concrete face rockfill dams [25-27]. However, the scarcity of observed data generally precludes the application of statistical analysis and machine learning in the study of dam deformation. In addition, the application of FEM requires accurate material parameters and reasonable assumptions of boundary conditions [28]. Some previous studies have conducted centrifugal model experiments to overcome the limitations of the aforementioned methods. Xu et al. [29] studied the influence of valley topography on the deformation of the dam and slab of the Hongjiadu concrete face rockfill dam through a centrifuge model test. Their results showed that the shape of the valley has a significant effect on stress and deformation of the slab. In addition, they found that increasing the filling density of the dam effectively reduced the stress on and deformation of the dam and slab. Excessive deformation of the dam and slab may result in the failure of a concrete face rockfill dam. The mechanisms responsible for the effects of valley topography on concrete face rockfill dams remain poorly understood. Therefore, there is a need for further extensive studies on the effects of valley topography on concrete face rockfill dams to facilitate effective risk prevention and control measures.

The present study established two concrete face rockfill dam centrifuge experimental models with different valley topographies. The deformation, stress, cracking process, and deflection modes of the concrete face rockfill dam models during the construction and impoundment stages were examined, and the influence of valley topography on the distributions of stress and cracks on the face slab were analyzed.

\section{Centrifuge Model}

2.1. Centrifuge Testing Program. The present study used the LXJ-4-450 geotechnical centrifuge in the China Institute of Water Resources and Hydropower Research (IWHR), which is shown in Figure 1. This centrifuge has a maximum rotation radius, maximum acceleration, payload, and maximum capacity of $5.03 \mathrm{~m}, 300 \mathrm{~g}, 1.5 \mathrm{ton}$, and $450 \mathrm{~g}$ ton $^{-1}$, respectively. The size of the test basket used was $1.5 \mathrm{~m} \times 1.0 \mathrm{~m} \times 1.5 \mathrm{~m}$. The maximum test model scaling ratio $N$ was set to 120 , and the maximum running acceleration during the experiment was $120 \mathrm{~g}$. A rigid model container with size dimensions of $1,350 \mathrm{~mm}$ (length) $\times 400 \mathrm{~mm}$ (width) $\times 900 \mathrm{~mm}$ (height) was used for the "U"-type valley dam model, whereas another with size dimensions of $1,280 \mathrm{~mm}$ (length) $\times 720 \mathrm{~mm}$ (width) $\times 940 \mathrm{~mm}$ (height) was used for the "V"-type valley dam model.

\subsection{Materials Used in the Centrifuge Model Experiments.} The filling material used in the centrifuge model experiments was consistent with that used in real-world concrete face rockfill dams. However, because of space constraints placed by the container, the size of particles used in the experiments was downscaled from those used in real-world dams. The experiments used in the present study utilized the mixed method to maintain hydraulic consistency with real-world concrete face rockfill dams as far as possible [30]. Figure 2 shows the particle size distribution of the rockfill of some existing sand-gravel dams compared to that of the experimental dam. Table 1 shows the parameters of the experimental dam material. Figure 3 shows samples of each material particle group after screening. 


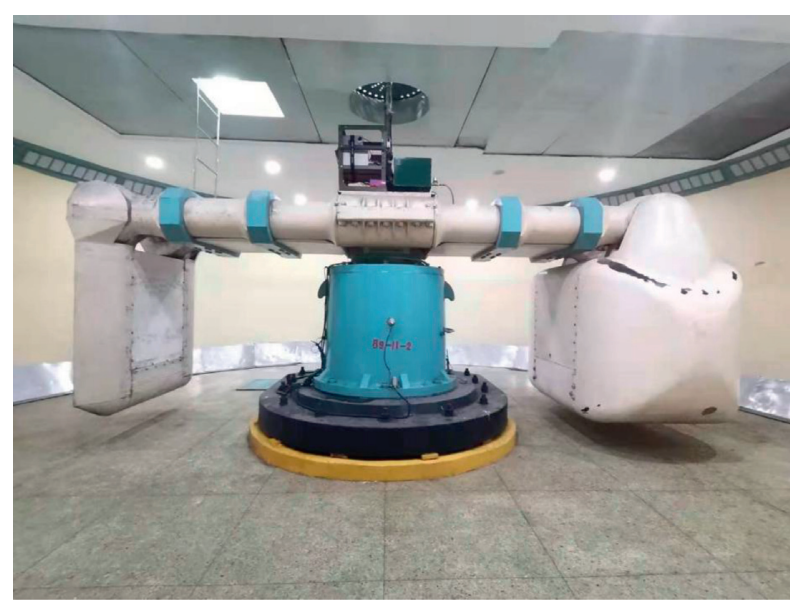

FIgUre 1: The centrifuge of the China Institute of Water Resources and Hydropower Research used in the present study.

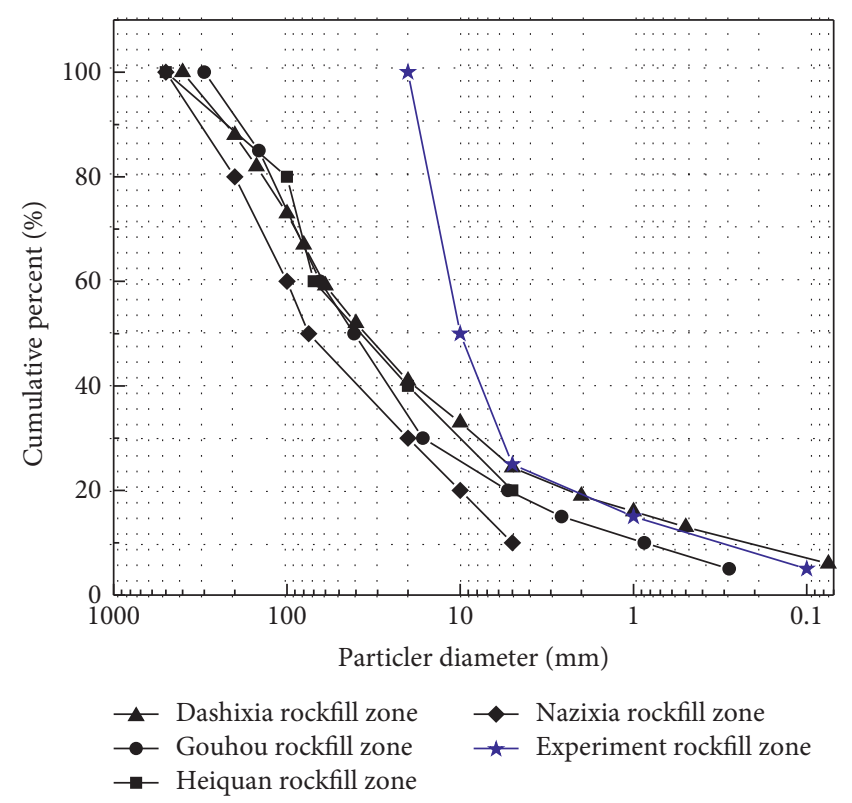

Figure 2: Particle size distribution of some existing concrete face rockfill dams compared to that of the experimental model dam used in the present study.

2.3. Model Preparation. The two concrete face rockfill dam models were established to analyze the behavior of a concrete face rockfill dam under the influence of " $U$ "-type and "V"-type valley topography models, respectively. The "U"-type valley topography model was used to examine the influence of wide and shallow valley topography on the behavior of a concrete face rockfill dam, whereas the "V"-type valley topography model was used to examine the influence of narrow valley topography. The "U"-type valley topography model had length, width, and height dimensions of $1,000 \mathrm{~mm} \times 720 \mathrm{~mm} \times 502 \mathrm{~mm}$, respectively, whereas those of the " $V$ "-type valley topography model were $400 \mathrm{~mm} \times 720 \mathrm{~mm} \times 502 \mathrm{~mm}$, respectively. Both models had a dam crest width of $40 \mathrm{~mm}$. Both dams had the same upstream and downstream slope inclines of $1: 1.5$ and $1: 1.4$, respectively. Since the focus of the experiment was on the upstream side of the dam and the concrete slabs, the downstream side of the dam was not represented in either of the two models. The "V"-type valley topography model used a trapezoidal valley topography with a top width, bottom width, and depth of $720 \mathrm{~mm}, 405 \mathrm{~mm}$, and $204 \mathrm{~mm}$, respectively. Figures 4 and 5 show the geometry and size of the models.

Since the thickness of a concrete face slab used for engineering application is typically $30 \mathrm{~cm}$ to $1 \mathrm{~m}$, it was difficult to scale the slab used in the model while retaining mechanical properties that are consistent with those in a real-world dam. To overcome this challenge, some researchers [31-33] have suggested that the slabs used in a centrifugal test of a face rockfill dam can be assumed to have only bending deformation. Therefore, the deflection $u$ of the thin slab can be expressed as

$$
u=\frac{\bar{M}}{E I}
$$

In equation (1), $\bar{M}=-\int\left[\int M(x) \mathrm{d} x\right] \mathrm{d} x+C_{1} x+C_{2}$, in which $M(x)$ is the bending moment of the thin plate section, $C_{1}$ and $C_{2}$ are the integral constants of boundary conditions, $E I$ is the bending stiffness of the thin plate, $E$ is the modulus of elasticity, and $I$ is the moment of inertia of the thin plate section. The values of $\bar{M}_{p}$ and $\bar{M}_{m}$ must satisfy the scaling laws since the external loads and boundary conditions borne by the prototype material and the model material of the thin plate are similar.

The bending stiffness of the model plate of prototype material and model material can be expressed as

$$
\begin{aligned}
& \bar{M}_{p}=E_{p} I_{p}=E_{p} \cdot \frac{d_{p}^{3}}{12} \cdot 1, \\
& \bar{M}_{m}=E_{m} I_{m}=E_{m} \cdot \frac{d_{m}^{3}}{12} \cdot 1 .
\end{aligned}
$$

In equations (2) and (3), $d_{p}$ and $d_{m}$ are the thicknesses of the face slab of the real-world dam prototype and the model, respectively, and $E_{p}$ and $E_{m}$ are the elastic modulus of the dam prototype and the model, respectively.

If the same material is in the prototype and model, the following holds true:

$$
\frac{\bar{M}_{p}}{\bar{M}_{m}}=\frac{E I_{p}}{E I_{m}}=\frac{(1 / 12) \cdot E \cdot d_{p}^{3}}{(1 / 12) \cdot E \cdot d_{m}^{3}}=N^{3} .
$$

In equation (4), $N$ is the scaling factor. The following can be obtained by substituting equations (2) and (3) into equation (4):

$$
d_{m}=\sqrt[3]{\frac{E_{p}}{E_{m}}} \frac{d_{p}}{N}
$$

The thickness of each layer of the plate used in the model should satisfy equation (5) to achieve bending stiffness consistent with real-world dam slabs.

Some studies have used a metal plate [31] or a plate composed of a composite resin material [32] to represent the slab in an experimental model since these materials have 
TABLE 1: Material parameters of dam zones used in the experimental model of the concrete face rockfill dam.

\begin{tabular}{lccccc}
\hline $\begin{array}{l}\text { Partition } \\
\text { material }\end{array}$ & $\begin{array}{c}\text { Dry density }(\mathrm{kg} \\
\left.\mathrm{m}^{-3}\right)\end{array}$ & $\begin{array}{c}\text { Maximum particle diameter } \\
(\mathrm{mm})\end{array}$ & $\begin{array}{c}\text { Median diameter } \\
(\mathrm{mm})\end{array}$ & $\begin{array}{c}\text { Nonuniform coefficient } \\
C_{u}\end{array}$ & $\begin{array}{c}\text { Curvature } \\
\text { coefficient } C_{c}\end{array}$ \\
\hline Rockfill & $1,850-2,005$ & 20 & 10 & 33.66 \\
\hline
\end{tabular}

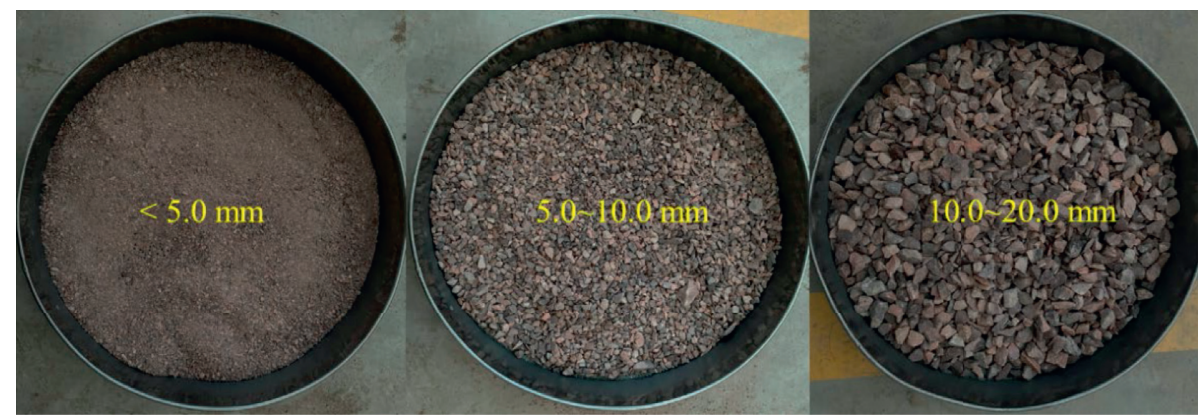

FIGURE 3: Particle group samples of material used in the experimental model of the concrete face rockfill dam used in the present study.

advantages, such as uniform material properties and ease of production. However, the cracking of the slab under a high load cannot be simulated using these materials due to their high strength and good ductility. The use of a cement mortar slab does allow the simulation of cracks. Thus, the present study used a cement mortar plate containing fiber material.

The elastic modulus of the concrete face slab used in the real-world prototype was $24 \mathrm{GPa}$, whereas its thickness was assumed to be $300 \mathrm{~mm}$. However, it is difficult to maintain the stability of a general cement mortar plate in centrifuge tests. Wang et al. [34] and Deng et al. [35] suggested that mixed fly ash or fiber can effectively enhance the strength of a cement mortar plate. The present study used a cement mortar plate composed of mixed fiber as according to Deng [35]. The thickness of the cement mortar model of the face slab was $8.0 \mathrm{~mm}$, whereas its elastic modulus was $21.6 \mathrm{GPa}$, as confirmed in the IWHR laboratory. The compressive and tensile strengths of the model face slab were $60 \mathrm{MPa}$ and $8 \mathrm{MPa}$, respectively, due to the addition of fibrous material to the cement mortar. In addition, a cement mortar toe wall was established in the experiment. Figure 6 shows photographs of the model face slab and the toe wall used in the present study.

\subsection{Model Construction, Instrumentation, and Testing} Procedure. The sidewalls of the container were covered with grease to minimize side friction. Furthermore, each dam model was constructed using five layers to maintain uniform densities. The heights of all layers from the first to fourth layer were $100 \mathrm{~mm}$, whereas the height of the fifth layer was $102 \mathrm{~mm}$. Each layer underwent 85\% relative compaction using a hand compactor at an approximate compaction energy of $750 \mathrm{kN} \cdot \mathrm{m}^{-3}$. Subsequent to the completion of the dam, a layer of sand with a thickness of $5 \mathrm{~mm}$ was deposited upstream, whereas the slabs were deposited upstream of the dam. Figure 7 shows a schematic diagram of the construction process.

Within the "U"-type valley topography model, eight pairs of half-bridge resistance strain gauges were attached on both sides of the slab at the main section of the dam, $165 \mathrm{~mm}$ away from the sidewall of the container. Strain gauges were placed at $100 \mathrm{~mm}$ intervals along with the slab. In addition, a strain gauge was placed $55 \mathrm{~mm}$ from the dam crest and another was positioned near the toe wall, thereby allowing the recording of the distributions of deformation and stress of the slab. Because of their limited number, only four pairs of strain gauges were attached on both sides of the face slab of the dam under the "V"-type valley topography model. The slab in which strain gauges were installed was $195 \mathrm{~mm}$ away from the sidewall of the container. Strain gauges were placed at $200 \mathrm{~mm}$ intervals along the slab, with an additional strain gauge placed $105 \mathrm{~mm}$ from the dam crest and another placed near the toe wall. In addition, one and four laser sensors were set at the dam crest and the upstream slope of each dam, respectively, to measure dam deformation. Figures 4 and 5 show the layout of the sensors for both dams, whereas Figure 8 shows both final dam models. Figure 9 shows the transducers used in the dam models, and Table 2 shows the transducer parameters.

The centrifugal model experiment was initiated when the dam model was fully prepared, and the present study examined dam deformation under four cases: (1) under a " $U$ "type valley topography model during the dam construction stage; (2) under a "U"-type valley topography model during the dam impound stage; (3) under a "V"-type valley topography during the model construction stage; (4) under a "V"-type valley topography during the dam impound stage. Table 3 shows the specific parameters of each case.

\section{Results}

3.1. Settlement of the Dam Crest. Figure 10 shows the settlement of the dam crest under both valley topography cases under increased centrifugal acceleration during the construction and impoundment stages. The settlement of the dam crest of both dams increased with increased centrifugal acceleration during the construction and impoundment stages. Within the "U"-type valley topography model, at a 


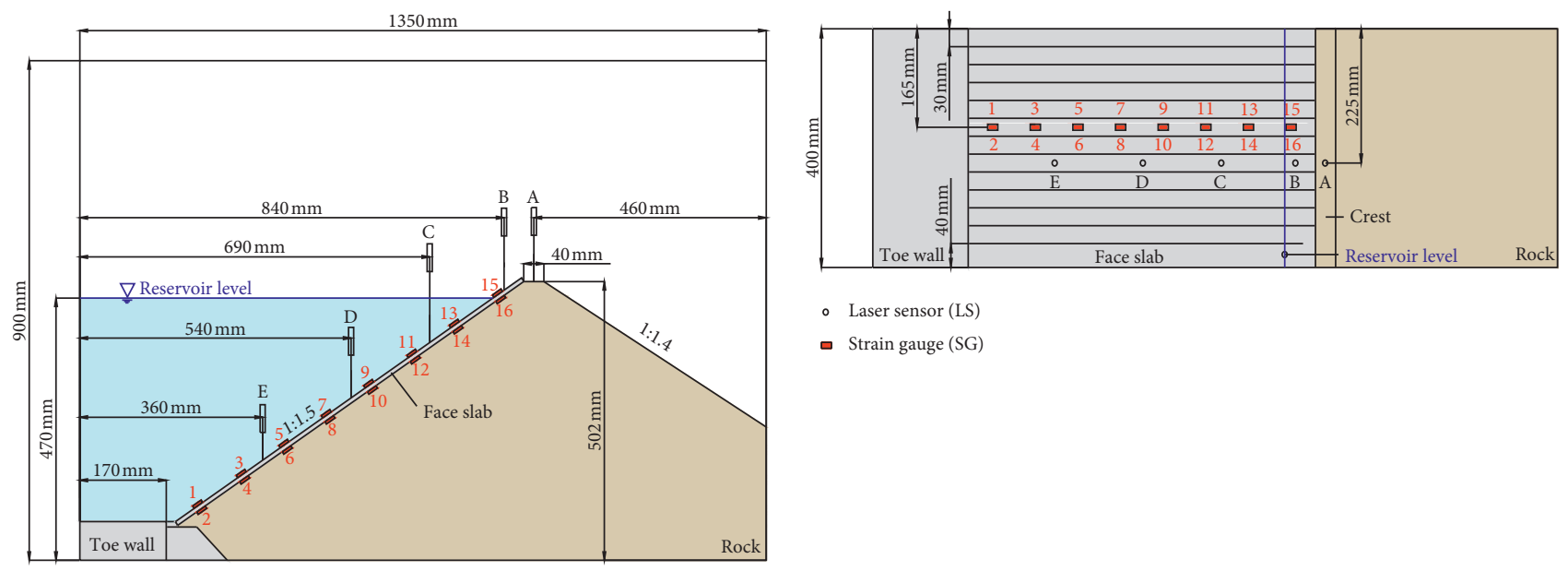

|

- Strain gauge $(\mathrm{SG})$

(a)

(b)

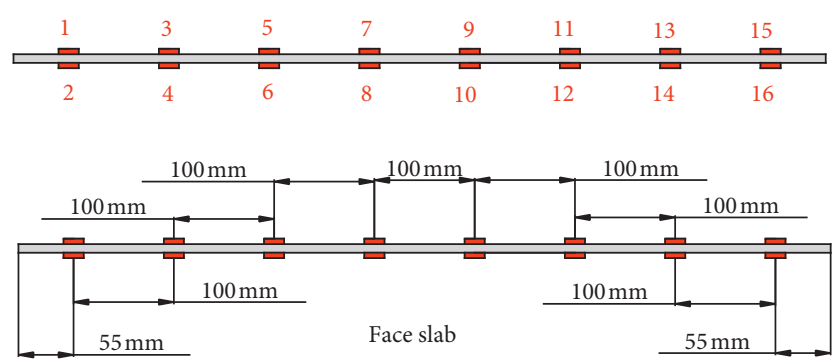

(c)

FIGURE 4: The layout scheme of the "U"-type dam model used in the current study: (a) side view of the dam, (b) plan view of the dam, and (c) the face slab.

centrifugal acceleration of $120 \mathrm{~g}$, the maximum dam crest settlement values during the construction and impoundment stages were $1.18 \mathrm{~mm}(0.23 \%$ of the dam height $)$ and $2.19 \mathrm{~mm}$ ( $0.43 \%$ of the dam height), respectively, whereas those in the "V"-type valley topography model were $2.53 \mathrm{~mm}(0.54 \%$ of dam height $)$ and $3.07 \mathrm{~mm}(0.61 \%$ of dam height), respectively. The maximum dam crest settlement in the "V"-type valley topography model exceeded that in the "U"-type valley topography model. This result could be attributed to interference by the valley model, with the compaction energy in the "U"-type model exceeding that in the "V"-type model. Therefore, the degree of compaction in the "U"-type valley topography model exceeded that in the "V"-type valley topography model. However, during the impoundment stage, the increments in the dam crest in the "U"-type and "V"-type valley topography models were $1.01 \mathrm{~mm}$ and $0.54 \mathrm{~mm}$, respectively. The increase in the settlement of the dam crest during the impoundment stage in the "V"-type valley topography model was $65 \%$ less than that of the "U"-type valley topography model. The increment of the settlement of the dam crest in the " $U$ "-type valley topography model exceeded that in the "V"-type valley topography model. This phenomenon suggested that the influence of impounded water pressure on the settlement of the dam crest in the " $U$ "-type valley topography exceeded that in the "V"-type valley topography model. This result could be attributed to the valley topography limiting the deformation of the dam, enhancing the comprehensive stiffness of the dam, and decreasing the increment in the settlement of the dam crest due to the pressure from the impounded water.

3.2. Displacement of Model Face Slabs. Figure 11 shows the vertical displacement of the upstream slope with increasing centrifugal acceleration from the construction to the impoundment stages in both the "U"-type and "V"type valley topography models. In the "U"-type valley topography model, displacement near the dam crest increased during the dam construction stage, whereas displacement at other positions on the upstream slope decreased. The final vertical displacements of B-E along the slope from the crest to the toe when centrifugal acceleration reached $120 \mathrm{~g}$ were $0.9 \mathrm{~mm},-3.1 \mathrm{~mm}$, $-3.75 \mathrm{~mm}$, and $-4.6 \mathrm{~mm}$, respectively. The vertical displacement of B during the impoundment stage decreased first, following which it slightly increased and finally stabilized. Only vertical displacement at B and C in the "V"-type valley topography model was recorded due to damage experienced by the laser transducers at D and 

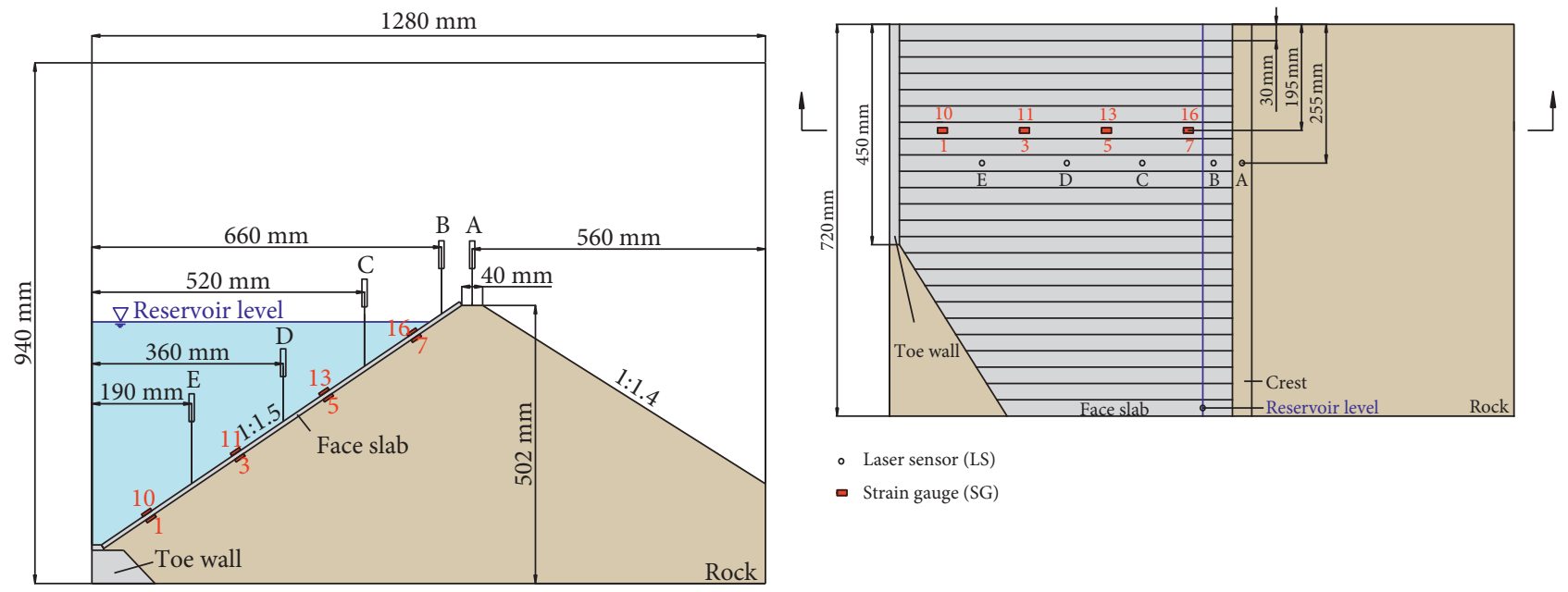

- Laser sensor (LS)

- Strain gauge (SG)

[ Laser sensor (LS)

- Strain gauge (SG)

(a)

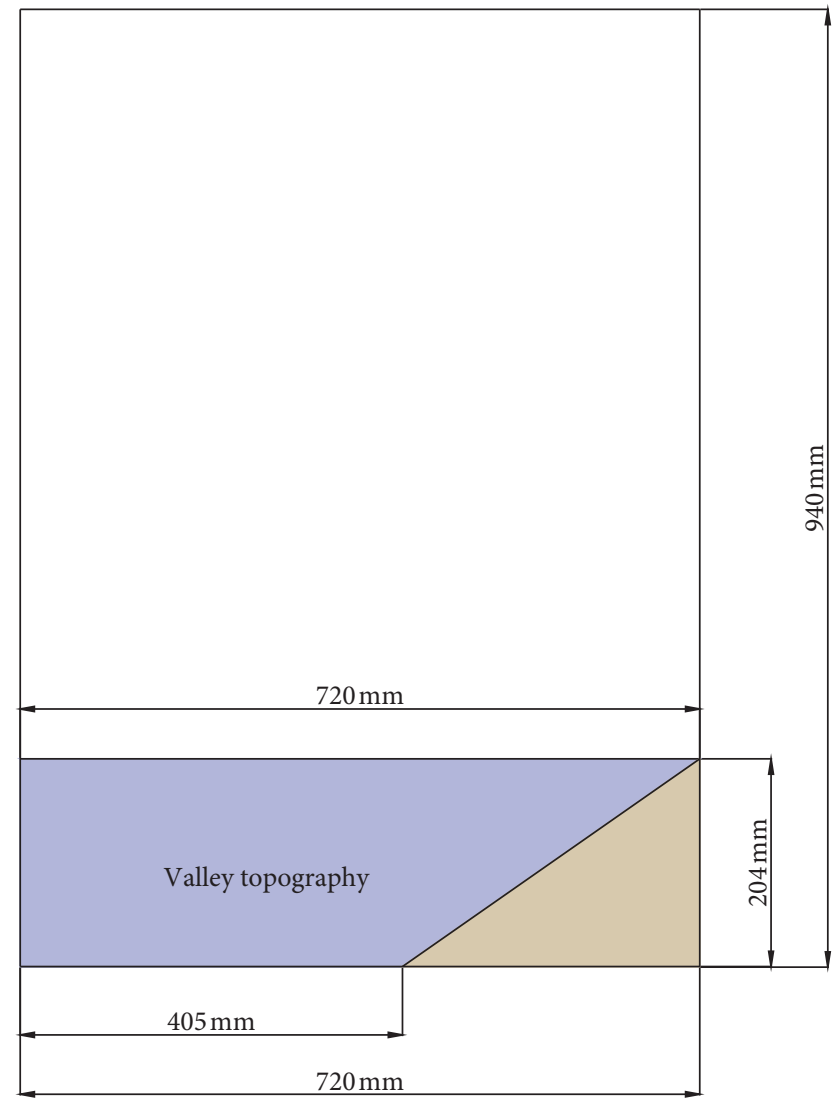

(c) (b)

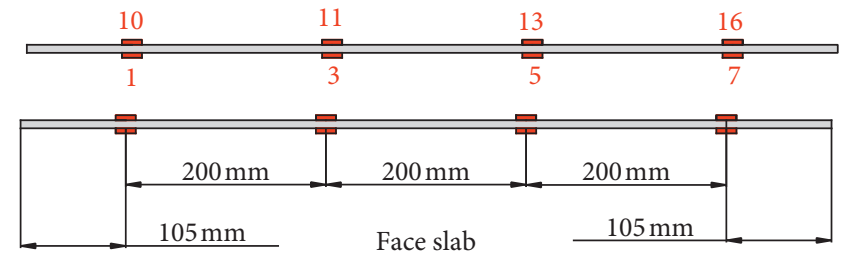

(d)

Figure 5: The layout scheme of the "V"-type dam model used in the current study: (a) side view of the dam, (b) plan view of the dam, (c) plan view of the valley topography, and (d) the face slab.

E. The vertical displacement at B during the construction stage increased near the crest as centrifugal acceleration increased. The displacement at $\mathrm{B}$ near the crest was $0.4 \mathrm{~mm}$ when centrifugal acceleration reached $120 \mathrm{~g}$, while that at $\mathrm{C}$ positioned in the center of the face slab was
$-0.6 \mathrm{~mm}$. The displacement of the slab near the dam crest decreased during the impoundment stage.

Both the "U"-type and "V"-type valley topography models experienced the same deformation of the upstream slope. The slab on the upstream slope near the dam crest 

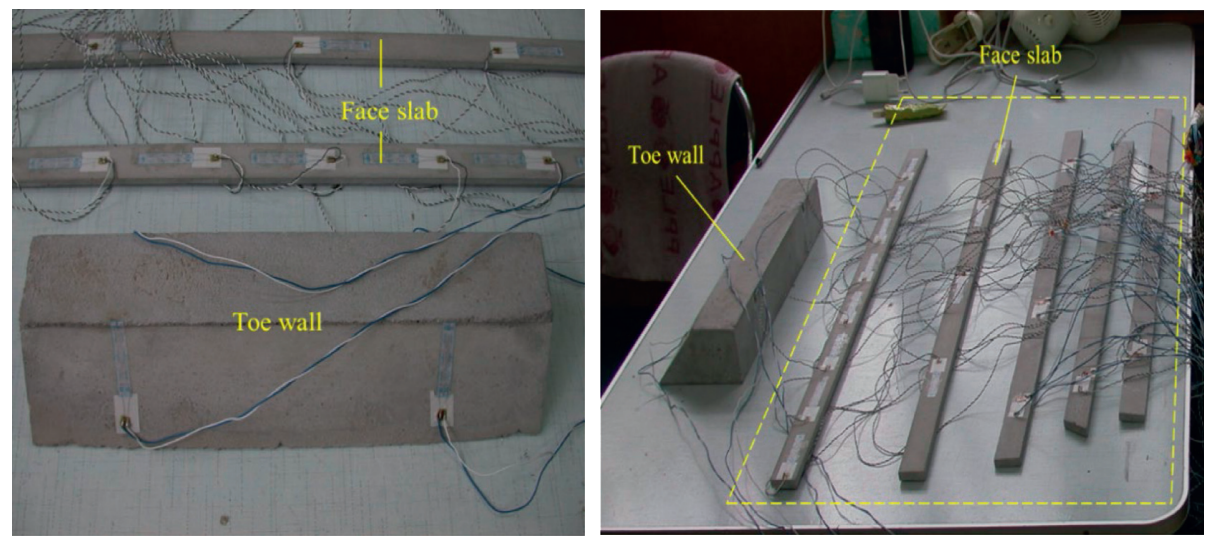

FIgURE 6: Photographs of the model face slab and the toe wall used in the present study.

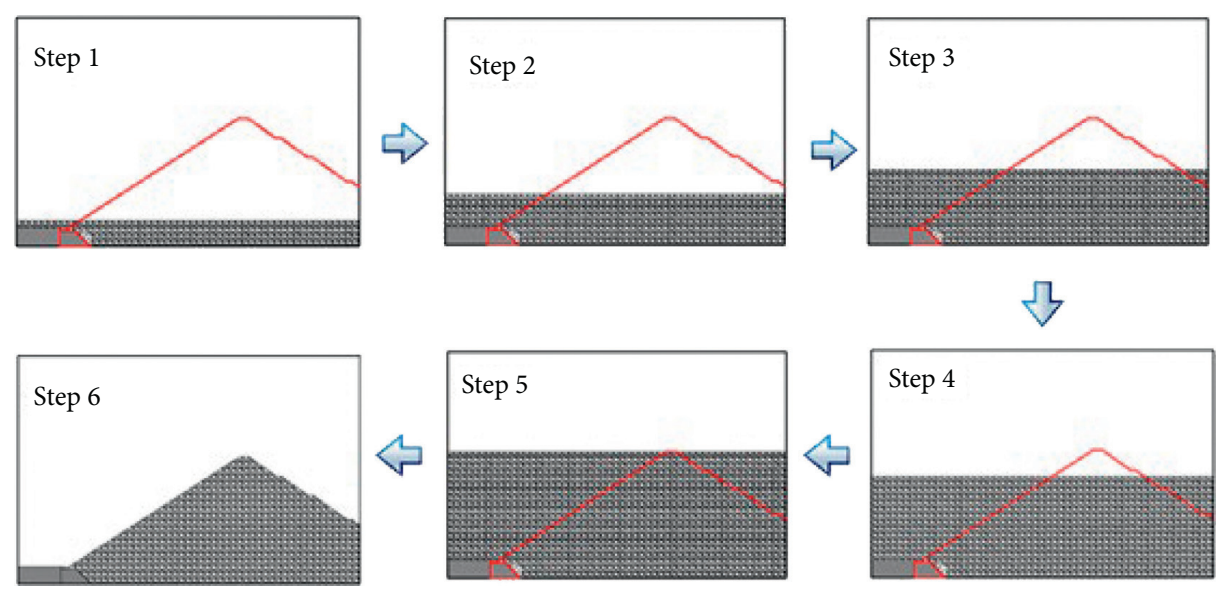

FIgURE 7: The filling process of each dam within the dam models used in the present study.

tended to move upstream during the construction stage, whereas those on the central and lower parts tended to move downstream. This result could be attributed to deformation of the dam crest resulting in lateral compression of the face slab and the movement of the face slab upstream. However, shrinkage of the center of the dam under its own weight resulted in displacement of the central part of the face slab and its movement downstream. During the impoundment stage, the slab near the dam crest moved downstream due to the pressure from the impounded water.

3.3. Induced Stresses in Model Face Slabs. During the construction stage, the slab moved towards the upstream side of the dam, resulting in pulling and compression of the upstream and downstream sides of the slab, respectively. During the impoundment stage, the slab deformed toward the downstream side of the dam due to the pressure of the impounded water, further resulting in compression and pulling of the upstream and downstream sides of the slab, respectively.

Figure 12 shows the distribution of stress of the face slab toward the upstream side of both the " $U$ "-type and "V"-type valley topography models during the construction and impoundment stages under a centrifugal acceleration of
$120 \mathrm{~g}$, in which a positive value represents tension and a negative value represents compression. Figure 12(a) shows the distribution of stress during the construction stage. Stress on the slab in the "U"-type valley topography model was mainly tensile stress. A maximum tensile stress of $5.2 \mathrm{MPa}$ occurred at $550 \mathrm{~mm}$ from the toe wall. Similar to that in the "U"-type valley topography model, tensile stress was the dominant form of stress on the slab in the "V"-type valley topography model, with the maximum tensile stress of $5.9 \mathrm{MPa}$ occurring $700 \mathrm{~mm}$ from the toe wall. Figure 12(b) shows the distribution of stress during the impoundment stage. The pressure from water impoundment during this stage resulted in the dominance of compressive stress on the slab. The maximum compressive stress in the " $U$ "-type valley topography model was $10.8 \mathrm{MPa}$, which occurs $355 \mathrm{~mm}$ from the toe wall, whereas that in the "V"-type valley topography model was $3.3 \mathrm{MPa}$ and occurred $500 \mathrm{~mm}$ from the toe wall.

Figure 13 shows the distribution of stress on the face slab toward the downstream side of the "U"-type valley topography model and "V"-type valley topography model during the construction and impoundment stages at a centrifugal acceleration of $120 \mathrm{~g}$. Figure 13(a) shows the distribution of stress during the construction stage. Compressive stress dominated in the "U"-type valley topography model, with a 

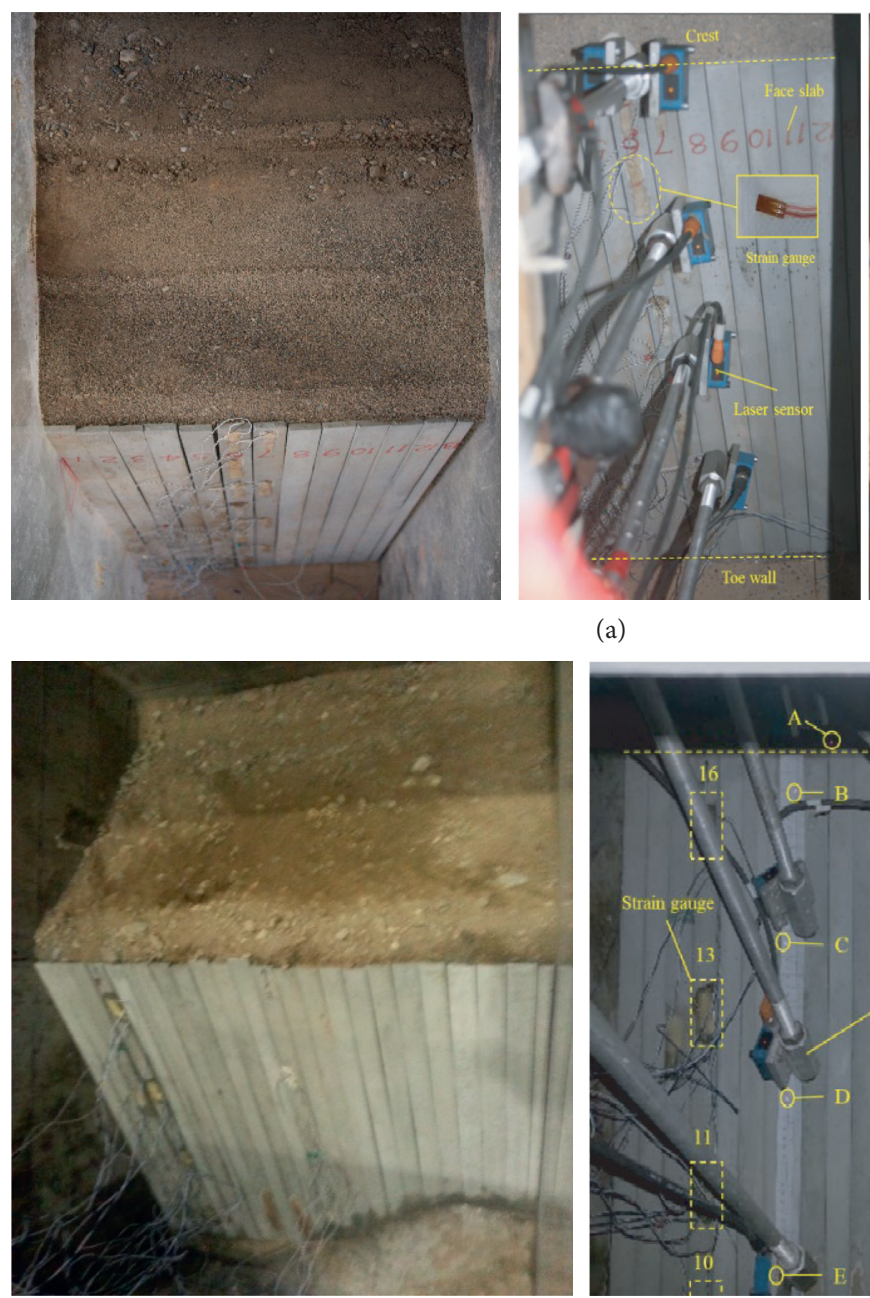

(a)
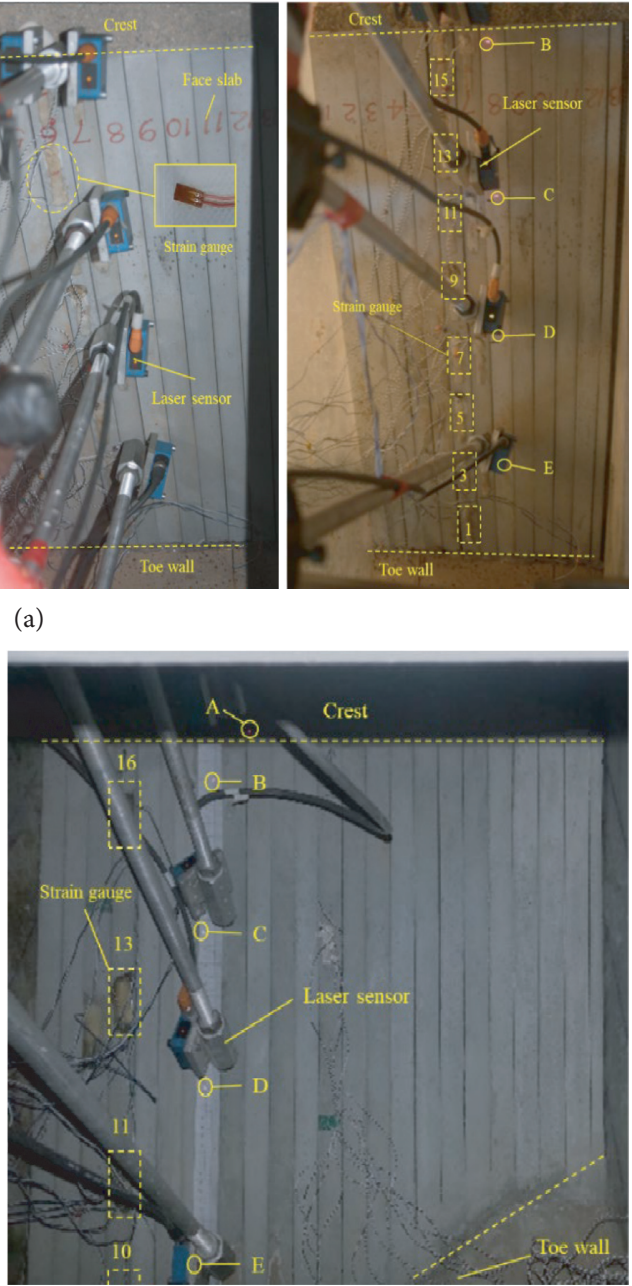

(b)

FIGURE 8: Photographs showing the final dam model used in the present study: (a) under the "U"-type valley topography and (b) under the "V"-type valley topography.

maximum compressive stress of $8.0 \mathrm{MPa}$ at $355 \mathrm{~mm}$ from the toe wall. The maximum compressive stress in the "V"-type valley topography model was $5.0 \mathrm{MPa}, 305 \mathrm{~mm}$ from the toe wall. Figure 13(b) shows the distribution of stress during the impoundment stage. Tensile stress dominated in the " $U$ "type valley topography model, with the maximum tensile stress of $7.5 \mathrm{MPa}$ occurring $555 \mathrm{~mm}$ from the toe wall. Tensile stress also dominated in the "V"-type valley topography model, with the maximum tensile stress of 6.5 MPa occurring $700 \mathrm{~mm}$ from the toe wall.

Surface stress changed from tensile stress (compressive stress) to compressive stress (tensile stress) from the construction stage to the impoundment stage. The distribution model of stress of the face slabs was the same for the " $U$ "type and "V"-type valley topography models, whereas the change in stress from the construction to the impoundment stages was different between the two models.

Figure 14 shows the distribution of the absolute difference in stress of the slab between the construction stage and the impoundment stage of each model under a centrifugal acceleration of $120 \mathrm{~g}$. The distribution of the change in stress along the slab on both sides showed a "D" shape in the " $U$ "-type valley topography model, whereas that in the "V"-type valley topography model showed a "B" shape. Figure 14(a) shows the distribution of the change in stress on the upstream side. In the "U"-type valley topography model, the maximum change in stress of $12.2 \mathrm{MPa}$ occurred $355 \mathrm{~mm}$ from the toe wall. However, the stress changed slightly near the toe wall and the dam crest by $2 \mathrm{MPa}$ and $1 \mathrm{MPa}$, respectively. The maximum change in stress in the "V"-type valley topography model of $8.6 \mathrm{MPa}$ occurred $305 \mathrm{~mm}$ from the toe wall. Figure 14(b) shows the distribution of the change in stress on the downstream side. In the " $U$ "-type valley topography model, the maximum change in stress of $11.5 \mathrm{MPa}$ occurred $555 \mathrm{~mm}$ from the toe wall. In the "V"-type valley topography model, the maximum changes in the stress of 8.7 MPa and 9.6 MPa occurred 305 and $705 \mathrm{~mm}$ from the toe wall, respectively. The maximum change in stress on the upstream side in the "V"-type valley topography model reduced by $31.2 \%$ compared to that in the " $U$ "type valley topography model. The maximum change in stress on the downstream side in the "V"-type valley 


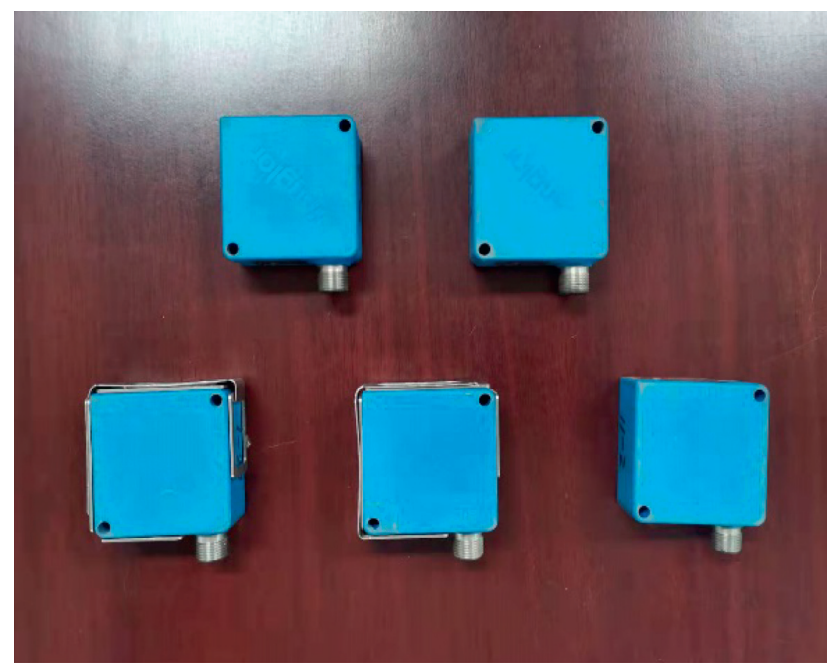

(a)

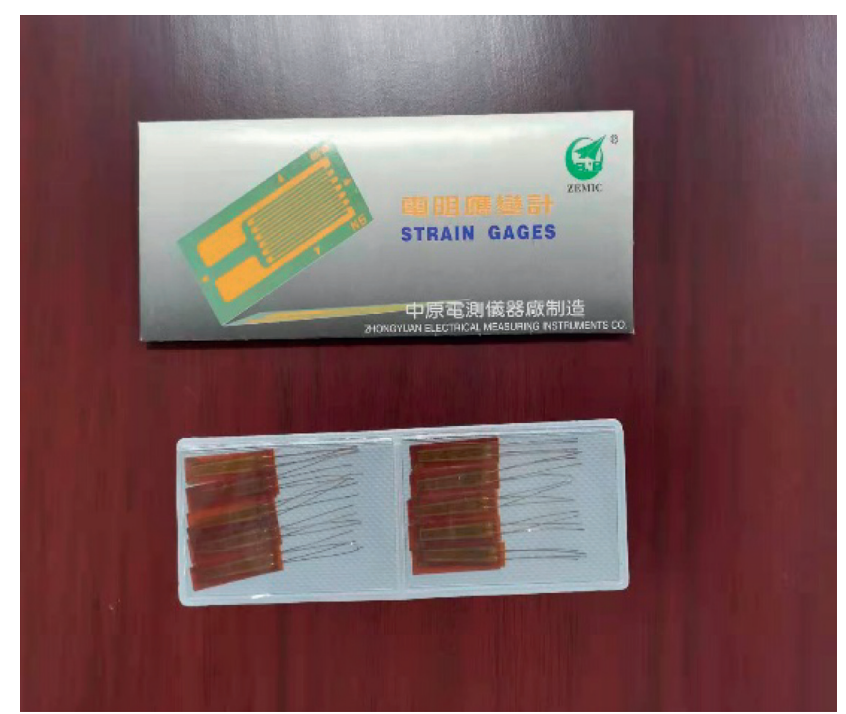

(b)

Figure 9: The transducers used in the dam experiment: (a) the laser transducers and (b) the strain gauges.

TABLe 2: The parameters of the transducer used in the dam experiment.

\begin{tabular}{lcccc}
\hline Parameters & Type & Range & Precision & Sensitivity ratio (\%) \\
\hline Laser transducers (LS) & WRANGLER & $50-100 \mathrm{~mm}$ & $5-20 \mu \mathrm{m}$ & $\sim$ \\
Strain gauge (SG) & BX120-3BA & $>10,000 \mu \varepsilon$ & $\sim$ & $2.06 \pm 1$ \\
\hline
\end{tabular}

TABLE 3: Experimental parameters of "V"-type and "U"-type experimental models of a concrete face rockfill dam used in the present study.

\begin{tabular}{|c|c|c|c|c|c|}
\hline Case & Target $g$ value & Dam height model (mm) & Dam height prototype (m & Valley topography type & Impoundment \\
\hline 1 & 120 & 502 & 60.24 & "U"-type & No \\
\hline 2 & 120 & 502 & 60.24 & "U"-type & Yes \\
\hline 3 & 120 & 502 & 60.24 & "V"-type & No \\
\hline 4 & 120 & 502 & 60.24 & "V"-type & Yes \\
\hline
\end{tabular}

topography model was reduced by $16.5 \%$ compared with that in the " $U$ "-type valley topography model.

The change in stress on the face slab during the impoundment process in the "U"-type valley topography model exceeded that in the "V"-type valley topography model. In addition, the change in stress in the center exceeded that near the dam crest and the toe in the "U"-type valley topography model, whereas the opposite pattern was observed in the "V"-type valley topography model. This difference could be attributed to the influence of the valley topography on the ability of the face slab to resist the pressure from the impounded water, further influencing the distribution of flexural deformation along the face slab. In the "U"-type valley topography model, the pressure of the impounded water and the deflection of the face slab was low near the dam crest. Although the pressure from the impounded water on the face slab was large near the toe wall due to the limited rockfill at this position, the stress on the face slab was small. However, the flexural deformation and stress of the face slab at the central height of the dam significantly exceeded that at other positions due to the large pressure from impounded water and the slight limit of the rockfill. In contrast to the situation in the "U"-type valley topography model, valley topography in the "V"-type valley topography model effectively constrained deformation at the central height of the rockfill, further resulting in less flexural deformation of the face slab near the central height compared to that near the crest and the toe wall.

3.4. Induced Stresses in the Model Face Slab before and after Cracking. The slab flexed during the impoundment stage, and the stress on the slab increased, eventually leading to cracking of the face slab. At this point, stress was instantly released at the crack, leading to an abrupt change to the stress curve. Figure 15 shows the stress variation curves of the slabs with the increased pressure from impounded water in both the "U"-type and "V"-type valley topography models. As shown in Figure 15(a), stress accumulated with increased pressure from impounded water in the " $U$ "-type valley topography model. Two obvious changes occurred when the pressure of impounded water increased at $500 \mathrm{~s}$ (30 g) and 2,000 s (90 g), with stress rapidly decreasing. As shown in Figure 15(b), only one abrupt change occurred in 


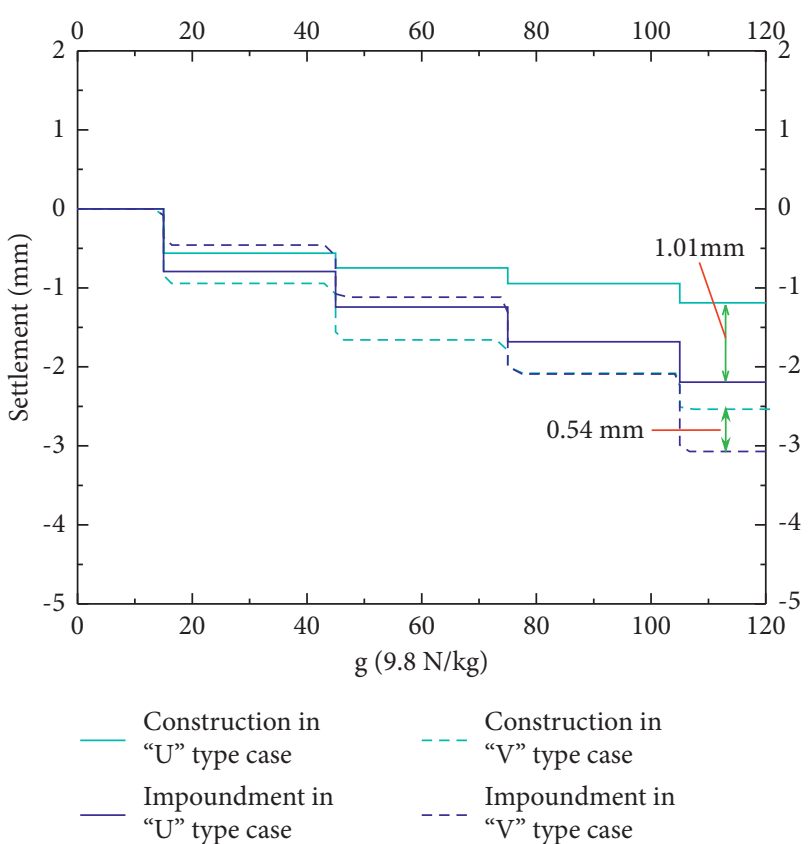

Figure 10: The settlement of the dam crest with increased centrifugal acceleration of both valley topography models used in the present study.

the "V"-type valley topography model at $500 \mathrm{~s}$ (30 g). This difference suggests that two cracking events occurred in the "U"-type valley topography model at $500 \mathrm{~s}$ (30 g) and 2,000 s $(90 \mathrm{~g})$, leading to the release of a large amount of stress. Furthermore, although a large amount of stress was released with the first cracking event, new stress was formed on the face slab with increasing pressure from impounded water, resulting in new cracking behavior. However, only one cracking event occurred in the "V"-type valley topography model at $500 \mathrm{~s}(30 \mathrm{~g})$ and no significant cracking event occurred subsequently.

Figure 16 shows the distribution of stress on both sides of the slab at the cracking events in the " $U$ "-type and "V"-type valley topography models. As shown in Figure 16(a), the crack event at $500 \mathrm{~s}$ ( $30 \mathrm{~g}$ ) on the upstream side in the "U"type valley topography model resulted in the maximum tensile stress decreasing from 3.8 MPa to $0.9 \mathrm{MPa}$ at $155 \mathrm{~mm}$ from the toe wall, whereas the maximum compressive stress on the downstream side of the slab decreased from $4.2 \mathrm{MPa}$ to $0.77 \mathrm{MPa}$ at the same position. However, no significant change in stress was observed at other locations. As shown in Figure 16(b), the maximum tensile stress on the upstream side decreased from 20.2 $\mathrm{MPa}$ to $12.5 \mathrm{MPa}$ at $355 \mathrm{~mm}$ from the toe wall when the next crack occurred at 2,000 s (90 g) in the " $U$ "-type valley topography model. In contrast, no change in stress occurred on the downstream side of the slab. This result could be attributed to damage to the strain gauge at $355 \mathrm{~mm}$ from the toe wall. Figure 16(c) shows the distribution of stress in the "V"-type valley topography model on both sides of the slab during the cracking event. Two obvious cracks occurred on the slab. The maximum compressive stress on the upstream side decreased from $3.5 \mathrm{MPa}$ to $0.8 \mathrm{MPa}$ at $105 \mathrm{~mm}$ from the toe wall. At the same time, compressive stress suddenly dropped from $6.0 \mathrm{MPa}$ to $0.45 \mathrm{MPa}$ at $505 \mathrm{~mm}$ from the toe wall. Tensile stress decreased from $5.52 \mathrm{MPa}$ to $1.15 \mathrm{MPa}$ at $105 \mathrm{~mm}$ from the toe wall on the downstream side, and tensile stress near the toe wall decreased from 5.1 MPa to 2.2 $\mathrm{MPa}$ during the cracking event at $505 \mathrm{~mm}$ from the toe wall. Similar to that observed in the "U"-type valley topography model, there was no significant change in stress at the uncracked position.

The "U"-type valley topography model experienced two cracks: one at the center and one at the lower part of the face slab. The crack near the toe wall occurred under a low impoundment pressure, whereas that at the central point of the face slab occurred under a high impoundment pressure. The two cracks in the "V"-type valley topography model were located near the toe wall and the dam crest, and the cracks occurred under the same impoundment pressure. Figure 17 shows the final state of the cracked slab for both the " $U$ "-type and "V"-type valley topography models. The difference in the position of the cracks between the two models could be attributed to the influence of valley topography on the deflection model of the face slab.

Deflection can be estimated by the strain on both sides of the face slab according to the methods proposed by [36-38]. Figure 18 shows the deflection model of the face slab during the cracking event. During the first crack of the slab in the " $U$ "-type valley topography model, the flexural deformation of the face slab towards the downstream side formed a " $D$ " shape, with the maximum deformation of $0.016 \mathrm{~mm} \mathrm{oc-}$ curring near the toe wall. The deflection of the slab further intensified during the second crack, and the position of the maximum deformation of $0.045 \mathrm{~mm}$ moved up to the center of the slab. The flexural deformation of the face slab towards the downstream side in the " $V$ "-type valley topography model formed a " $\mathrm{B}$ " shape. During the cracking of the face slab, the maximum deformation of $0.041 \mathrm{~mm}$ and $0.055 \mathrm{~mm}$ occurred near the toe wall and the dam crest, respectively.

There was one maximum deformation position during the deformation process of the face slab in the " $U$ "-type valley topography model, whereas there were two positions in the "V"-type valley topography model. This difference could be attributed to the valley topography limiting the deformation of the rockfill at the central height of the dam, thereby improving comprehensive stiffness and further enhancing resistance to pressure from impounded water at this position. Therefore, the deformation of the center of the slab in the "V"-type valley topography model under pressure from impounded water decreased, while that near the toe wall and the dam crest increased. Furthermore, the stress of the face slab near the dam crest and toe wall increased, with cracks subsequently occurring nearby.

\section{Discussion}

The current study established two experimental concrete face rockfill dam models. The experimental models established in the current study differ from those of existing dam studies due to their following characteristics: (1) a geotechnical centrifuge was used to examine deformation of the dam and slab during the construction and impoundment 


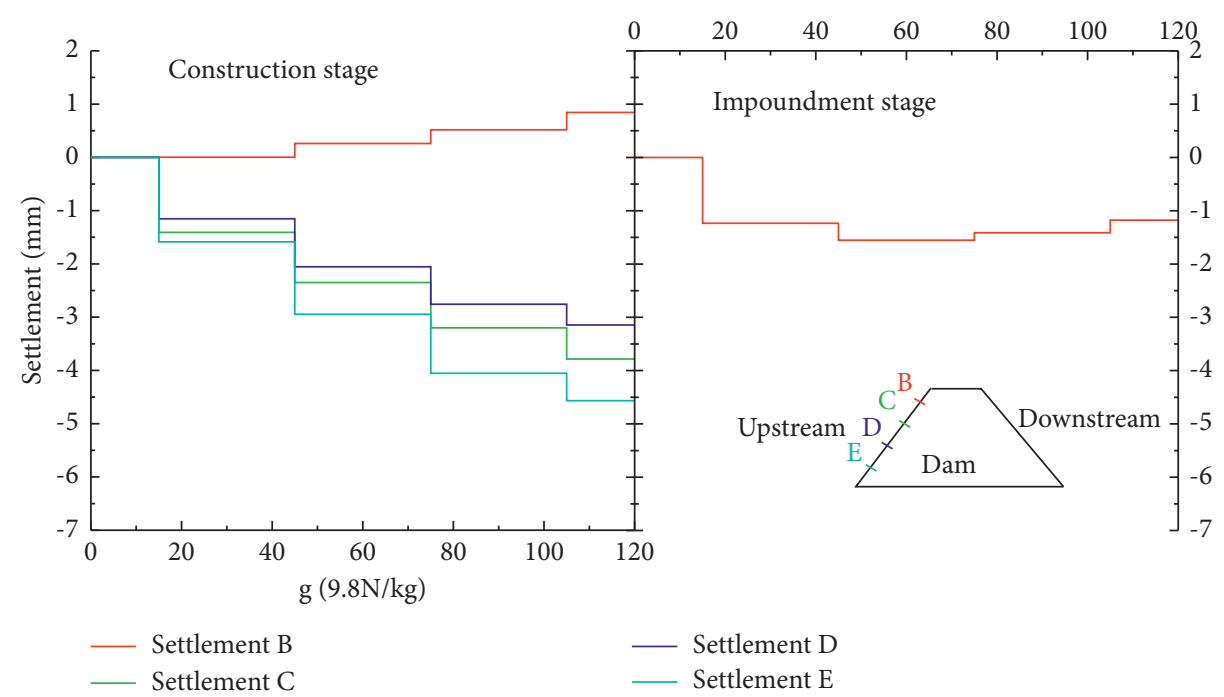

(a)

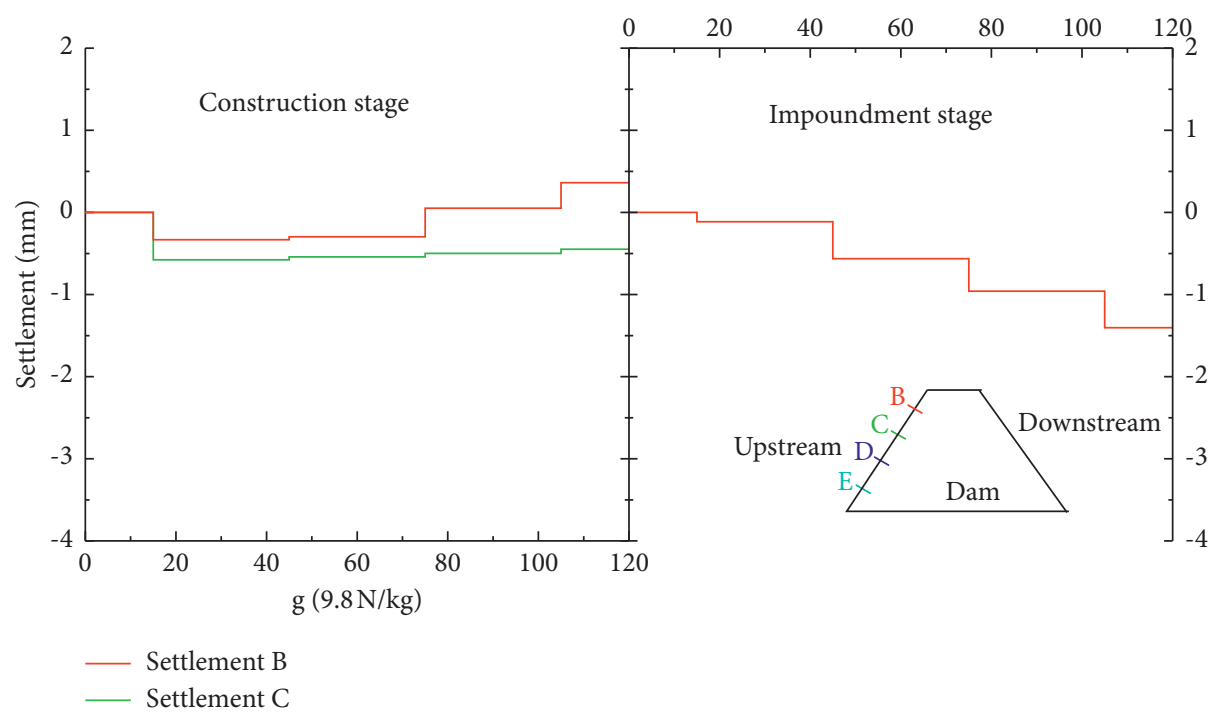

(b)

Figure 11: Vertical displacement along the face slab with increased centrifugal acceleration in the "U"-type and the "V"-type valley topography models: (a) the "U"-type valley topography model and (b) the "V"-type valley topography model.

stages. The resulting deformation of the dam and the stress on the face slab were consistent with those of a real-world dam. (2) A concrete valley was established to investigate the characteristics of deformation and stress of the dam model in the "V"-type valley topography model. (3) A cement mortar panel was installed in both models to allow the process of cracking of the face slab during the impoundment stage to be observed.

Numerous studies have reported the deformation and stress characteristics of a dam during the construction and impoundment stages, thereby allowing a comparison with the result of the present study. The upstream impoundment increased settlement of the dam crest and enhanced stress on the face slab. Some cracks occurred at the central height of the dam in the "U"-type valley topography model and near the toe wall and the dam crest in the "V"-type valley topography model during the impoundment stage. This result is consistent with phenomena observed for concrete face rockfill dams [12-15]. However, the cracks in the slab in the "U"-type valley topography model occurred sequentially with increasing pressure from impounded water, whereas those in the "V"-type valley topography model occurred under the same pressure from impounded water. These phenomena have not been previously reported and could be attributed to the assumption of continuity of the face slab material, which would result in an inability to observe the entire process of the face slab failure during the cracking event $[12,14,16,17,20]$. 


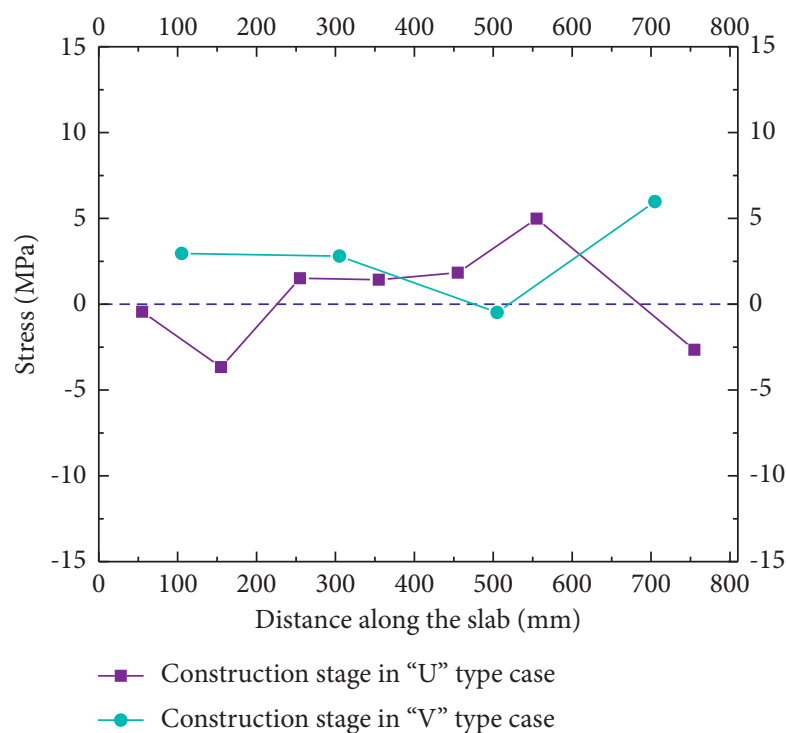

(a)

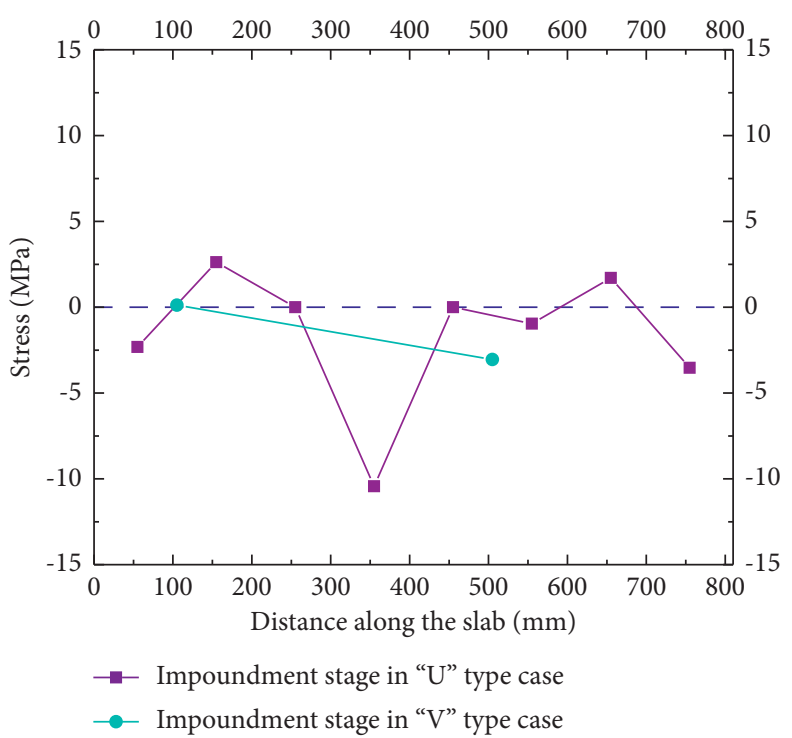

(b)

FIGURE 12: The distribution of stress on the upstream side of the slab in both valley topography models under a centrifugal acceleration of $120 \mathrm{~g}$ : (a) the construction stage and (b) the impoundment stage.

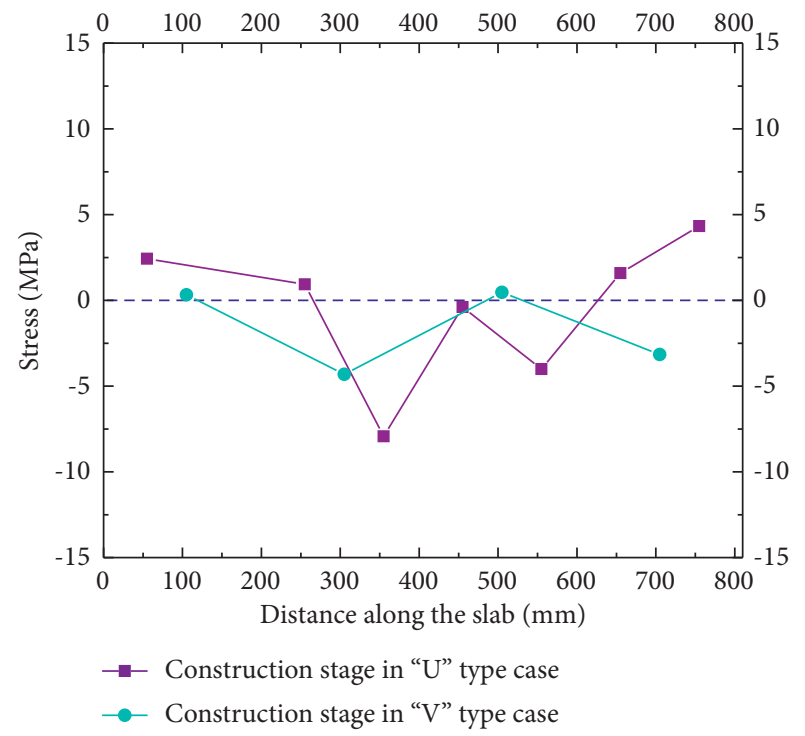

(a)

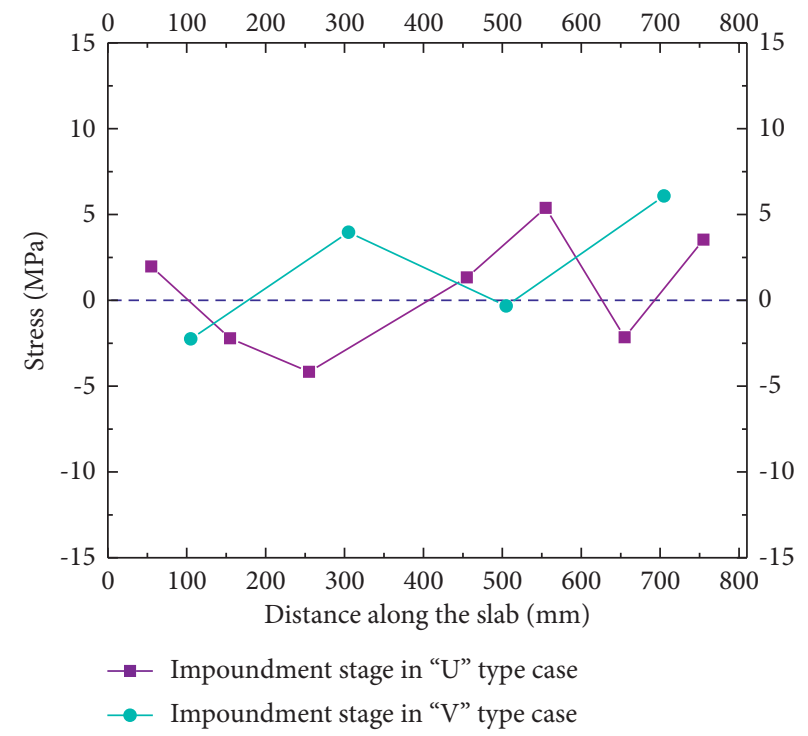

(b)

FIgUre 13: The distribution of stress on the downstream side of the dam slab in both valley topography models under a centrifugal acceleration of $120 \mathrm{~g}$ : (a) the construction stage and (b) the impoundment stage. 


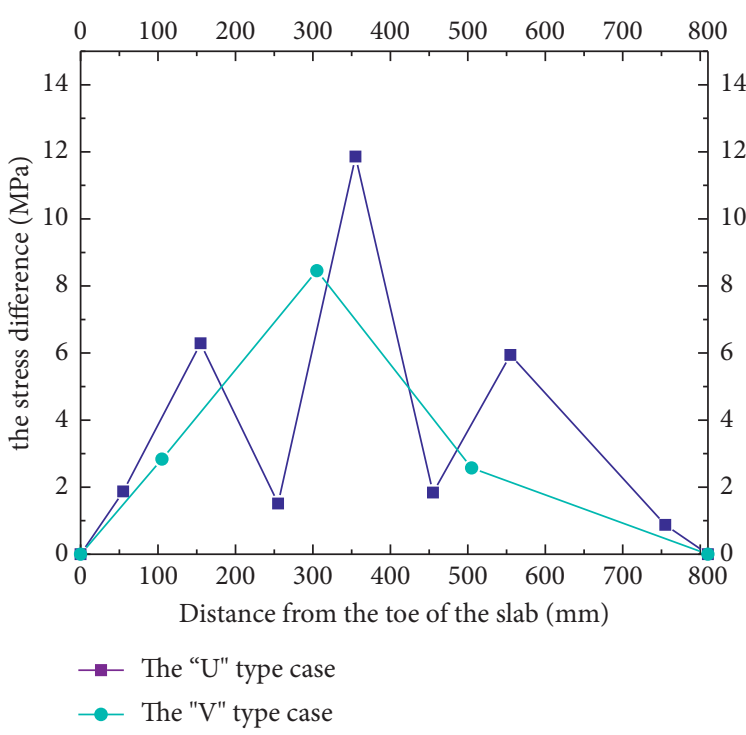

(a)

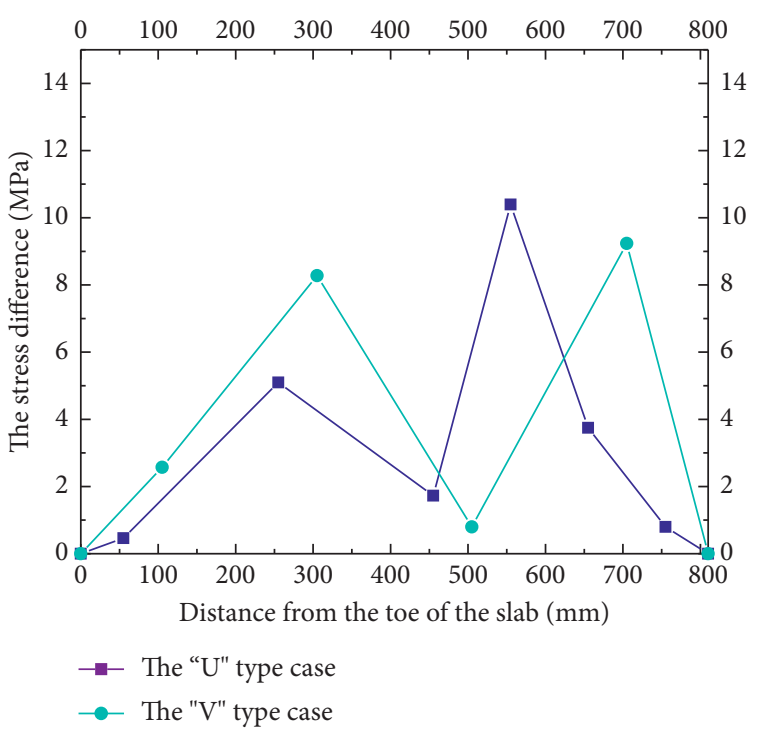

(b)

FIGURE 14: The distribution of stress on the face slab under a centrifugal acceleration of $120 \mathrm{~g}$ in the "U"-type and "V"-type valley topography model dams: (a) on the upstream side and (b) on the downstream side.

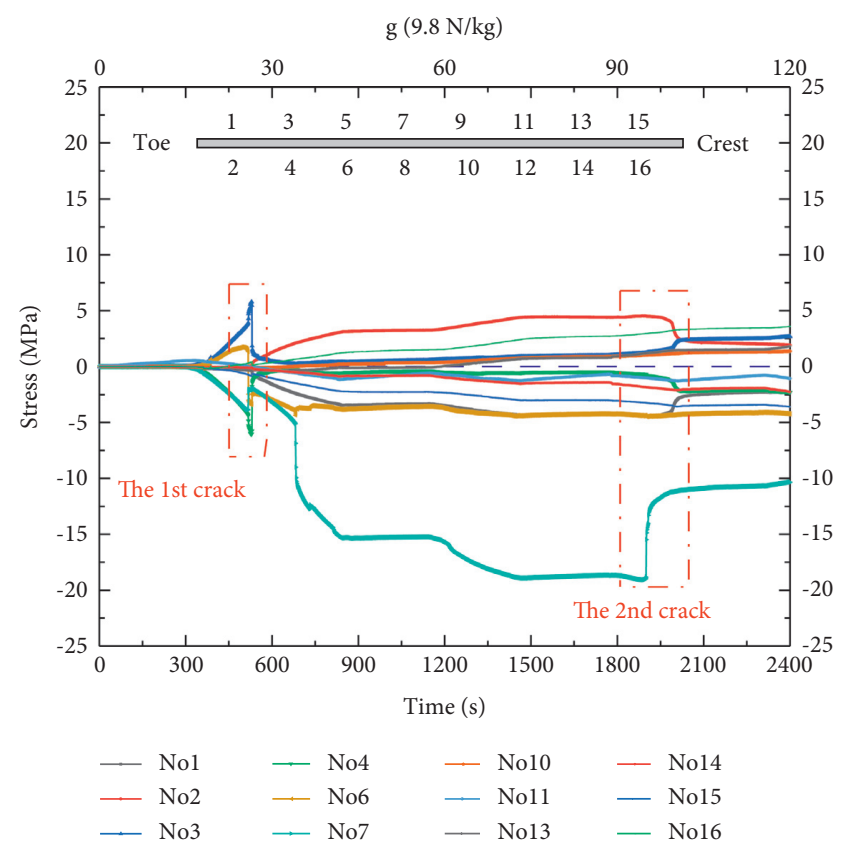

(a)

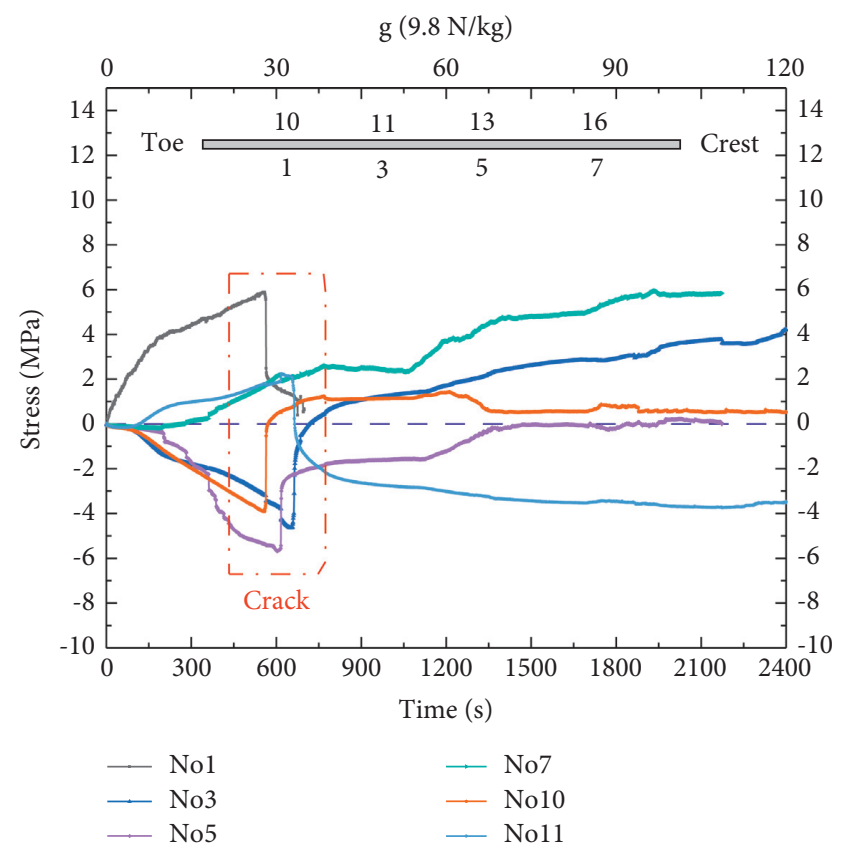

(b)

FIGURE 15: Stress experienced by the slab of a "U"-type and "V"-type valley topography model with increasing pressure due to impounded water: (a) the "U"-type case and (b) the "V"-type case. 

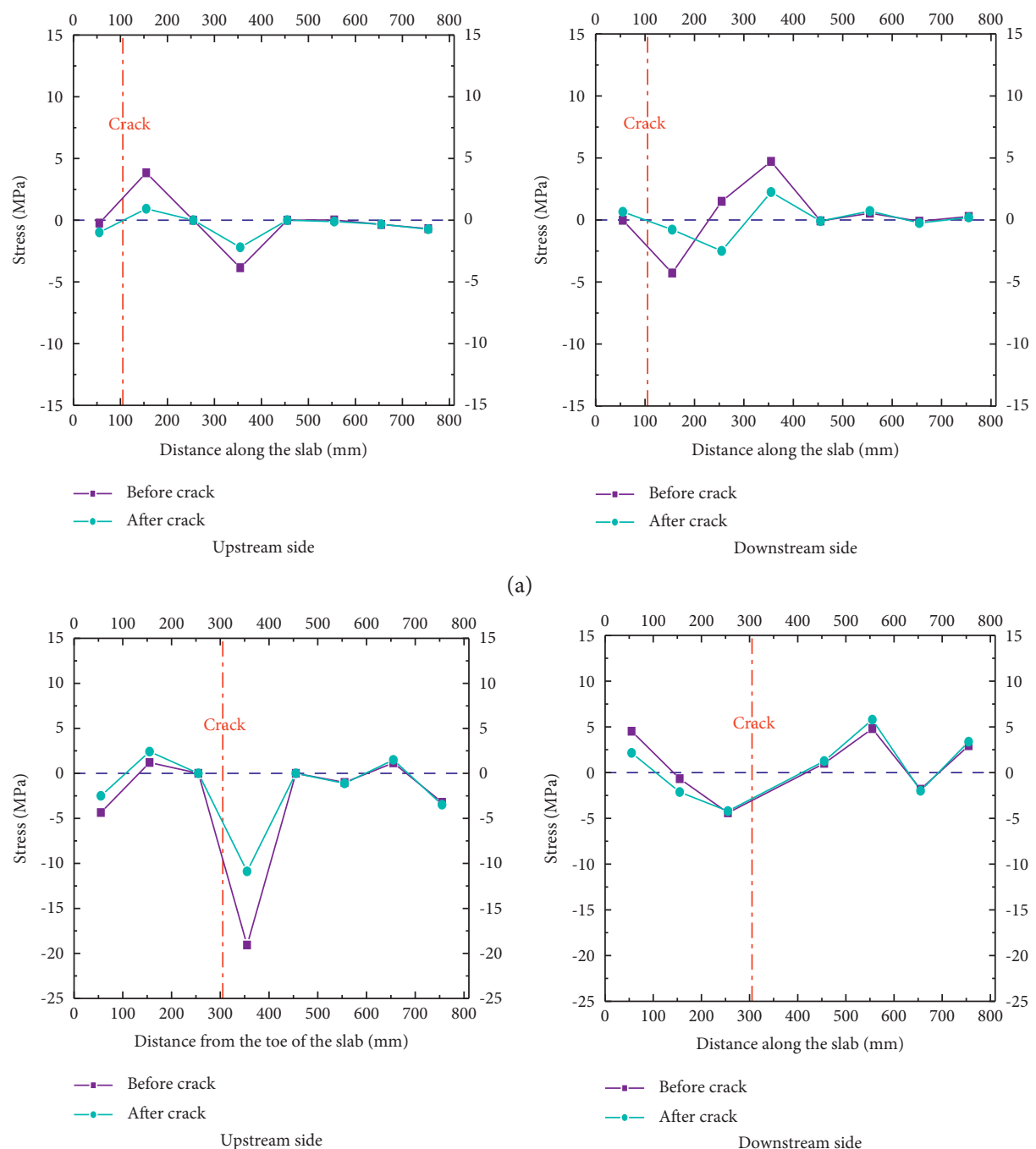

(b)
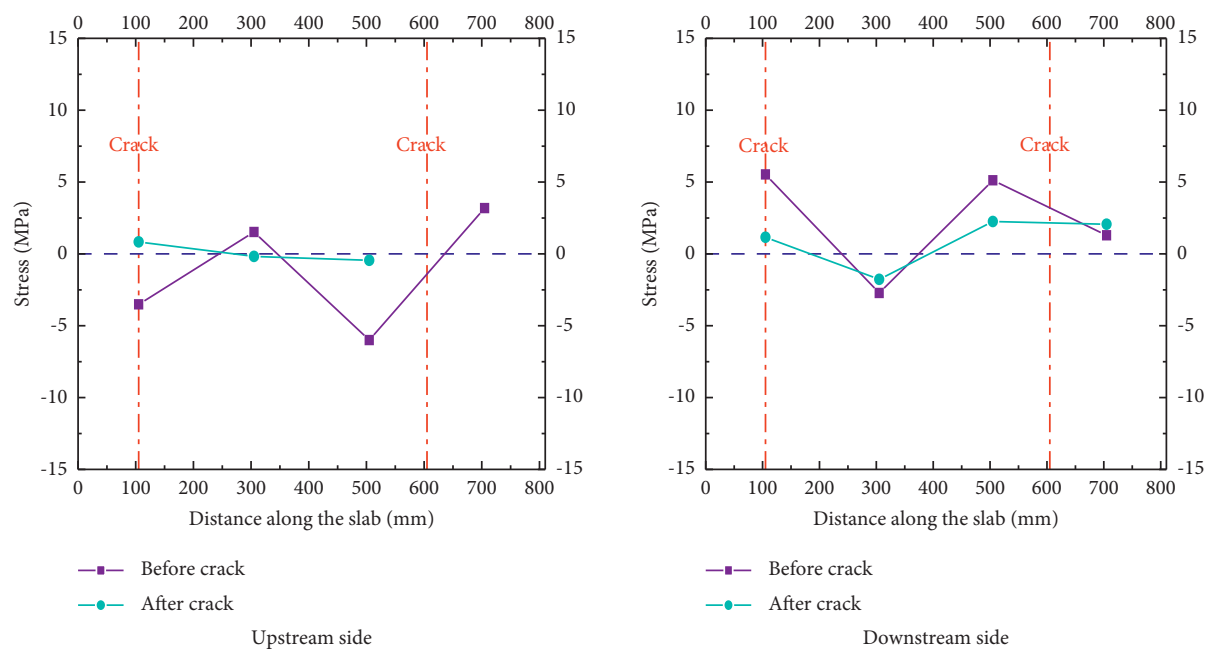

(c)

FIGURE 16: The distribution of stress on the slab before and after cracking in both types of valley topography models: (a) at 500-600 s in the "U"-type model, (b) at 1,800-2,000 s in the "U"-type model, and (c) at 500-600 s in the "V"-type model. 


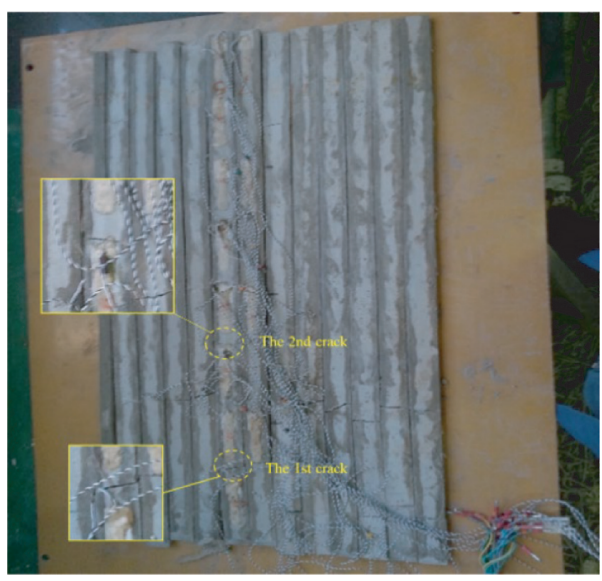

(a)

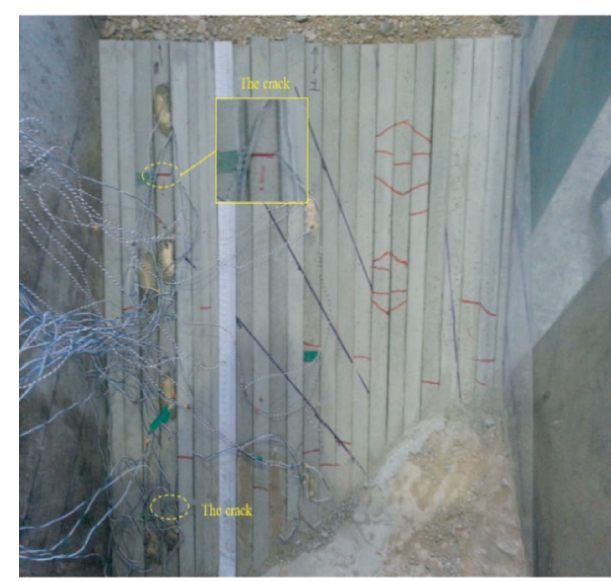

(b)

FIGURE 17: The final state of the cracked slabs in both types of valley topography models: (a) in the "U"-type model and (b) in the "V"-type model.

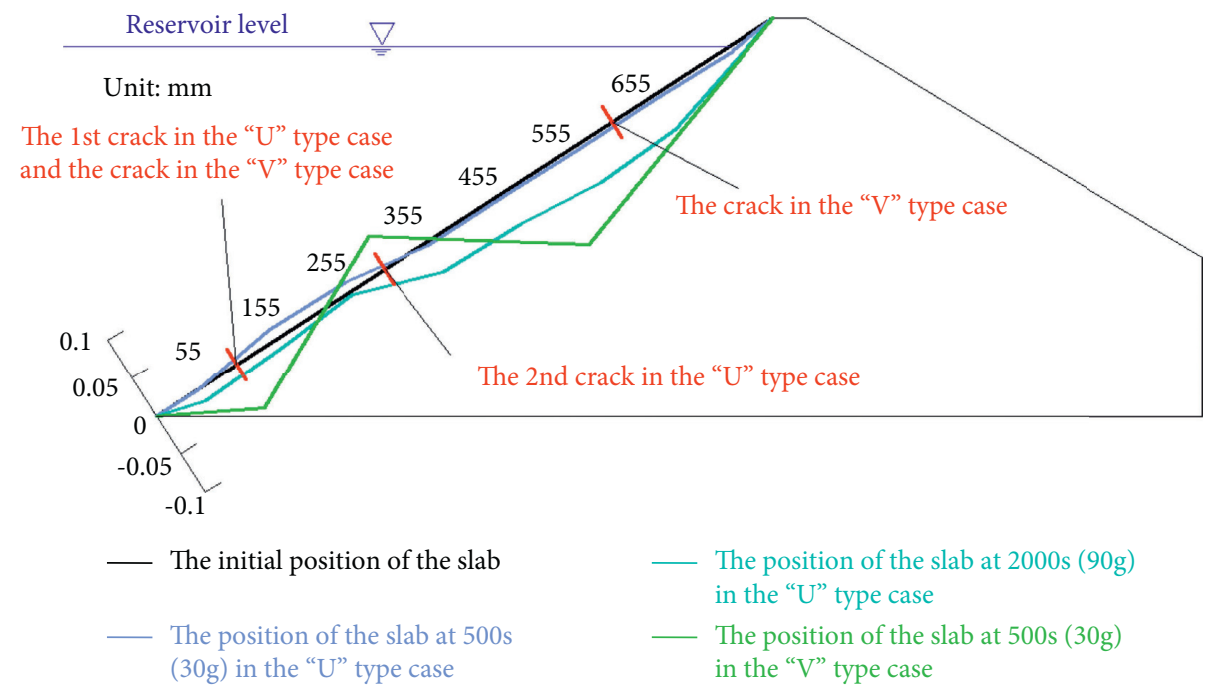

FIGURE 18: The deformation of the slab before cracking events in the " $U$ "-type and " $V$ "-type valley topography models.

\section{Conclusions}

The present study conducted two concrete face rockfill dam experiments to examine the effect of valley topography on the behavior of a dam from the construction to the impoundment stages. A particular focus of the present study was on the influence of valley topography on the behavior of the dam. The conclusions drawn are summarized below.

Valley topography had no significant impact on the deformational behavior of the concrete face rockfill dam from the construction to the impoundment stages. Settlement of the dam crest increased during the construction stage and the upstream dam slope moved under pressure to the upstream side. At this time, the face slab was flexed to the upstream side. The settlement of the dam crest further increased during the impoundment stage, the upstream slope of the dam began to move downstream under pressure, and the slab flexed to the downstream side. The deflection of the face slab from the construction to the impoundment stages changed from the upstream side to the downstream side, and the surface stress of the face slab changed from tensile stress (compressive stress) to compressive stress (tensile stress).

The "V"-type valley topography effectively reduced the increments in dam crest settlement and the stress on the slab resulting from pressure of impounded water. The increase in the settlement of the dam crest in the "V"-type valley topography model during the impoundment stage decreased by $65 \%$ compared to that in the "U"-type valley topography model. In addition, the change in stress in the "V"-type valley topography model from the construction to the impoundment stages was reduced by $16.5 \%$ compared with that in the "U"-type valley topography model. The influence of the pressure from impounded water on settlement of the dam crest and face stress in the "U"-type valley topography model exceeded that in the "V"-type valley topography model.

Valley topography significantly affected the mode of cracking and the location of the face slab. The cracks in the face 
slab in the "U"-type valley topography model were located in the central and lower part of the face slab. Cracks near the toe wall occurred under a low pressure from impounded water, whereas the crack in the center occurred under a higher pressure. However, the cracks in the face slab in the "V"-type valley topography model were located near the toe wall and the top of the dam and occurred under the same pressure of impounded water.

Valley topography influenced the cracking mode and the location of the face slab by influencing the flexural model of the face slab. After impoundment, the deformation of the entire face slab in the "U"-type valley topography model took on a " $D$ " shape, and only one extreme point formed during the impoundment stage. The flexural deformation of the face slab in the "V"-type valley topography model took on a "B" shape, and two extreme points of flexural deformation were formed.

\section{Data Availability}

The data used to support the findings of this study are available from the corresponding author upon request.

\section{Conflicts of Interest}

The authors declare that they have no conflicts of interest.

\section{Acknowledgments}

The authors from Xi'an University of Technology and the China Institute of Water Resources and Hydropower Research prepared this study with the financial support of the National Funds for Distinguished Young Youths (Grant no. 52125904), the National Natural Science Foundation of China (Grant nos. 51979224 and 51909215), the China Postdoctoral Science Foundation (Program nos. 2021T140554 and 2020M683527), the National Natural Science Foundation for Young Scientists of China (Grant no. 51809290), and the Natural Science Basic Research Program of Shaanxi (Program no. 2020JQ-641).

\section{References}

[1] N. M. Mahabad, R. Imam, Y. Javanmardi, and H. Jalali, "Three-dimensional analysis of a concrete-face rockfill dam," Proceedings of the Institution of Civil Engineers - Geotechnical Engineering, vol. 167, no. 4, pp. 323-343, 2014.

[2] M. Wang, J. Chen, and W. Xiao, "Experimental and numerical comparative study on gravity dam-reservoir coupling system," KSCE Journal of Civil Engineering, vol. 22, no. 10, pp. 3980-3987, 2018.

[3] D. Zou, H. Han, H. I. Ling, Y. Zhou, and J. Liu, "An approach for the real-time slip deformation coupled with strain softening of a high rockfill dam subjected to pulse-like ground motions," Soil Dynamics and Earthquake Engineering, vol. 117, pp. 30-46, 2019.

[4] I. D. Kirn and G. S. Sarkaria, "Influence of canyon shape on the design of concrete dams," Civil Engineering and Public Works Review, Lomax, Erskine and Co., London, UK, 1955.
[5] H. H. Thomas, The Engineering of Large Dams, John Wiley \& Sons, NY, U S.A, 1976.

[6] N. L. S. Pinto and P. L. Marques, "Estimating the maximum face deflection in CFRDs," Hydropower Dams, vol. 5, no. 6, pp. 28-30, 1998.

[7] S. Giudici, R. Herweynen, and P. Quinlan, "HEC experience in concrete faced rockfill damsPast, present and future," in Proceedings of the international symposium on concrete faced rockfill dams, pp. 29-46, Beijing, China, September 2000.

[8] P. Johannesson, "Design improvements of high CFRDs constructed of low modulus rock," in Proceedings of the Symposium on CFRD-Dams Honoring, Florianópolis, Brazil, October 2007.

[9] Y.-S. Kim and B.-T. Kim, "Prediction of relative crest settlement of concrete-faced rockfill dams analyzed using an artificial neural network model," Computers and Geotechnics, vol. 35, no. 3, pp. 313-322, 2008.

[10] D. Znamensky, Valleys Shape Influence on the Volume Safety Factor and Arch Effect of High CFR Recently Built in brazil, Commission Internationale des Grands Barrages Brasilia, Paris, France, 2009.

[11] R. Sukkarak, P. Jongpradist, and P. Pramthawee, "A modified valley shape factor for the estimation of rockfill dam settlement," Computers and Geotechnics, vol. 108, pp. 244-256, 2019.

[12] Y. Arici, "Behaviour of the reinforced concrete face slabs of concrete faced rockfill dams during impounding," Structure and Infrastructure Engineering, vol. 9, no. 9, pp. 877-890, 2013.

[13] S. Cheng, G. Zhang, and J. Zhang, "Effect of foundation topography on the stress-displacement response of concrete faced rockfill dam," Journal of Hydroelectric Engineering, vol. 2008, no. 5, pp. 53-58, 2008, (in chinese).

[14] Y. Arici and H. F. Zel, "Comparison of 2D versus 3D modeling approaches for the analysis of the concrete faced rock-fill Cokal Dam," Earthquake Engineering \& Structural Dynamics, vol. 42, 2013.

[15] F. N. Dang, C. Yang, and H. B. Xue, "The effect of valley topography on deformation properties of CFRD," Journal of Hydraulic Engineering, vol. 39, no. 4, pp. 435-442, 2014, (in chinese).

[16] J. Yang, G. Y. Li, and T. Shen, "Stress-deformation properties of super-high CFRDs under complex terrain conditions," Chinese Journal of Geotechnical Engineering, vol. 36, no. 4, pp. 775-781, 2014.

[17] R. J. Wang, W. Liu, and L. U. Zhi-Nan, "Effect of valley width/ height ratio on stress deformation of rock-fill dam," Journal of Northwest Forestry University, vol. 42, no. 8, (in chinese), 2014.

[18] M. Karalar and M. Çavuşli, "Evaluation of 3D nonlinear earthquake behaviour of the Ilısu CFR dam under far-fault ground motions," Advances in Civil Engineering, vol. 2019, Article ID 7358710, 15 pages, 2019.

[19] M. Karalar and M. Çavuşli, "Effect of normal and shear interaction stiffnesses on three-dimensional viscoplastic creep behaviour of a CFR dam," Advances in Civil Engineering, vol. 2018, Article ID 2491652, 17 pages, 2018.

[20] Y. Gao, H. Liu, and M. S. Won, "Behavior of rockfill dam under complex terrain condition," Arabian Journal of Geosciences, vol. 13, no. 19, 2020.

[21] Y. Li, Y. Li, Y. Li, and Z. Liu, "Effect of silane coupling agent on improving adhesive property between acidic aggregate and hydraulic asphalt," Journal of Materials in Civil Engineering, vol. 33, no. 7, 2021. 
[22] Y. Li, H. Zhang, L. Wen, and N. Shi, “A prediction model for deformation behavior of concrete face rockfill dams based on the threshold regression method," Arabian Journal for Science and Engineering, vol. 46, no. 6, pp. 5801-5816, 2021.

[23] L. Wen, Y. Li, and J. Chai, "Multiple nonlinear regression models for predicting deformation behavior of concrete-face rockfill dams," International Journal of Geomechanics, vol. 21, no. 2, Article ID 04020253, 2021.

[24] L. Wen, Y. Yang, and Y. Li, "A comprehensive evaluation method of safety behavior of concrete face rockfill dam based on regression relationship method and fuzzy recognition model," IOP Conference Series: Earth and Environmental Science, vol. 643, no. 1, Article ID 012070, 2021.

[25] C. Soydemir and B. Kjaernsli, "Deformations of membranefaced rockfill dams," in Proceedings of the 7th European Conference on Soil Mechanics and Foundation Engineering, vol. 3, pp. 281-284, Brighton, England, September 1979.

[26] R. P. Clements, "Post-construction deformation of rockfill dams," Journal of Geotechnical Engineering, vol. 110, no. 7, pp. 821-840, 1984.

[27] Z. Li, "Defirmation observation on Longxi concrete face rockfill dam," in Proceedings of the International Symposium on High Earth-Rockfill Dams, pp. 514-521, Beijing, China, October 1993.

[28] M. W. Seo, I. S. Ha, and Y. S. Kim, "Behavior of concrete-faced rockfill dams during initial impoundment," J. Journal of Geotechnical and Geoenvironmental Engineering, vol. 135, no. 8, pp. 1070-1081, 2015.

[29] Z. P. Xu, Y. Shao, and B. X. Hu, "Study on stress and deformation properties of high concrete face rock-fill dam in narrow valley," Water Resources and Hydropower Engineering, vol. 39, 2005.

[30] The ministry of water Resources of the people's Republic of China, Specification of soil experiment (SL 237-1999), China Water and Power Press, Beijing, China, (in Chinese), 1999.

[31] A. N. Schofield, "Dynamic and earthquake geotechnical centrifuge modeling," in Proceedings of the international conference on Recent Advances in Geotechnical Engineering and Soil Dynamics, Cambridge University, Cambridge, UK, April 1981.

[32] M.-K. Kim, S.-H. Lee, Y. W. Choo, and D.-S. Kim, "Seismic behaviors of earth-core and concrete-faced rock-fill dams by dynamic centrifuge tests," Soil Dynamics and Earthquake Engineering, vol. 31, no. 11, pp. 1579-1593, 2011.

[33] R. N. Taylor, Centrifuges in Modeling: Principles and Scale Effects, Taylor and Francis Group, Oxfordshire, UK, 2018.

[34] L. Wang, F. Guo, Y. Lin, H. Yang, and S. W. Tang, "Comparison between the effects of phosphorous slag and fly ash on the C-S-H structure, long-term hydration heat and volume deformation of cement-based materials," Construction and Building Materials, vol. 250, Article ID 118807, 2020.

[35] F. Deng, Y. Chi, L. Xu, L. Huang, and X. Hu, "Constitutive behavior of hybrid fiber reinforced concrete subject to uniaxial cyclic tension: experimental study and analytical modeling," Construction and Building Materials, vol. 295, no. 4, Article ID 123650, 2021.

[36] S. H. Kim, J. J. Lee, and I. B. Kwon, "Structural monitoring of a bending beam using Brillouin distributed optical fiber sensors," Smart Materials and Structures, vol. 11, no. 3, pp. 396-403, 2002.

[37] Z. Dan, "Distributed optical fiber sensor based on botdr and its application to structural health monitoring," China Civil Engineering Journal, vol. 36, no. 11, pp. 83-87, 2003, (in Chinese).
[38] H. Sui, B. Shi, and D. Zhang, "Study on distributed optical fiber sensor-based monitoring for slope engineering," Chinese Journal of Rock Mechanics and Engineering, vol. 27, pp. 3725-3731, 2008, (in Chinese). 